\title{
On the Spectrum of the Reduced Wave Operator with Cylindrical Discontinuity*
}

\author{
Willi Jäger \\ Department of Mathematics \\ The University of Heidelberg \\ D-69120 Heidelberg 1 \\ Germany \\ and \\ Yoshimi Saitō \\ Department of Mathematics \\ University of Alabama at Birmingham \\ Birmingham, Alabama 35294 \\ U. S. A.
}

*) This work was supported by Deutche Forschungs Gemeinschaft through SFB 359. 


\section{$\S 1$. Introduction.}

Consider the differential expression

$$
h=-\mu(x)^{-1} \Delta .
$$

Here $\Delta$ is the Laplacian in $\mathbf{R}^{N}$ with $N \geq 2$, and $\mu(x)$ is a positive function on $\mathbf{R}^{N}$ given by

$$
\mu(x)= \begin{cases}\mu_{1} & \left(x \in \Omega_{1}\right), \\ \mu_{2} & \left(x \in \Omega_{2}\right),\end{cases}
$$

where $\mu_{1}, \mu_{2}>0, \mu_{1} \neq \mu_{2}$, and $\Omega_{\ell}, \ell=1,2$, are open sets of $\mathbf{R}^{N}$ such that

$$
\left\{\begin{array}{l}
\Omega_{1} \cap \Omega_{2}=\emptyset, \\
\overline{\Omega_{1}} \cup \Omega_{2}=\Omega_{1} \cup \overline{\Omega_{2}}=\mathbf{R}^{N},
\end{array}\right.
$$

$\overline{\Omega_{\ell}}$ being the closure of $\Omega_{\ell}$. It is easy to see that a selfadjoint realization $H$ of $h$ is given by

$$
\left\{\begin{array}{l}
D(H)=H^{2}\left(\mathbf{R}^{N}\right), \\
H u=h u
\end{array}\right.
$$

in the Hilbert space

$$
X=L_{2}\left(\mathbf{R}^{N} ; \mu(x) d x\right),
$$

where $D(H)$ denotes the domain of $H, H^{k}\left(\mathbf{R}^{N}\right)$ denotes the $k$-th order Sobolev space over $\mathbf{R}^{N}$, and $h u$ should be taken in the sense of distributions. The separating surface $S$ is defined by

$$
S=\overline{\Omega_{1}} \cap \overline{\Omega_{2}}=\partial \Omega_{1}=\partial \Omega_{2}
$$

$\partial \Omega_{\ell}, \ell=1,2$, being the boundary of $\Omega_{\ell}$. Let

$$
n^{(\ell)}(x)=\left(n_{1}^{(\ell)}(x), n_{2}^{(\ell)}(x), \cdots, n_{N}^{(\ell)}(x)\right) \quad(\ell=1,2)
$$

be the unit outward normal of $\Omega_{\ell}$ at $x \in \partial \Omega_{\ell}=S$. Obviously we have $n^{(1)}(x)+n^{(2)}(x)=0$ for $x \in S$. Eidus [6] considered the operator $H$ under the following assumptions: there exist positive constants $c_{1}$ and $c_{2}$ such that

$$
\left|n_{N}^{(1)}(x)\right| \geq c_{1} \quad(x \in S)
$$

and

$$
\left|x \cdot n^{(1)}(x)\right| \leq c_{2} \quad(x \in S),
$$

where $x \cdot n^{(1)}(x)$ is the inner product of $x$ and $n^{(1)}(x)$ in $\mathbf{R}^{N}$. Note that a cone having its vertex at the origin and the positive $x_{N}$-axis as its axis satisfies 
(1.8) and (1.9). Imposing the above assumptions, Eidus [6] proved the limiting absorption principle for $H$, that is, by denoting by $R(z)$ the resolvent of $H$, the limits

$$
\lim _{\eta \downarrow 0} R(\lambda \pm i \eta)=R_{ \pm}(\lambda) \quad \text { in } \mathbf{B}\left(L_{2,1}\left(\mathbf{R}^{N}\right), L_{2,-1}\left(\mathbf{R}^{N}\right)\right)
$$

exist for $\lambda>0$, where the weighted $L_{2}$ space $L_{2, t}\left(\mathbf{R}^{N}\right), t \in \mathbf{R}$, is defined by

$$
L_{2, t}\left(\mathbf{R}^{N}\right)=\left\{f:(1+|x|)^{t} f(x) \in L_{2}\left(\mathbf{R}^{N}\right)\right\},
$$

and $\mathbf{B}(X, Y)$ is the Banach space of all bounded linear operators from $X$ into $Y$. Then, Saito [13] showed that $L_{2,1}\left(\mathbf{R}^{N}\right)$ and $L_{2,-1}\left(\mathbf{R}^{N}\right)$ in (1.10) can be replaced by $L_{2, \delta}\left(\mathbf{R}^{N}\right)$ and $L_{2,-\delta}\left(\mathbf{R}^{N}\right)$ with $\delta>1 / 2$, respectively. This means that the limiting absorption principle for $H$ holds on the same weighted $L_{2}$ spaces as are used for the Schrödinger operator (cf. Agmon[1], IkebeSaitō [7] and Saitō [11]). Then Roach-Zhang [10] has shown that $u=R^{ \pm}(\lambda) f$, where $\lambda>0$ and $f \in L_{2, \delta}\left(\mathbf{R}^{N}\right)$ with $\delta>1 / 2$, is characterized as a unique solution of the equation

$$
\left(-\mu(x)^{-1} \Delta-\lambda\right) u=f
$$

with the radiation condition

$$
\lim _{R \rightarrow \infty} \frac{1}{R} \int_{B_{R}}|\nabla u \mp i \sqrt{\lambda \mu(x)} \widetilde{x} u|^{2} d x=0 \quad\left(\widetilde{x}=\frac{x}{|x|}\right),
$$

$B_{R}$ being the ball with radius $R$ and center at the origin. The condition (1.13) is a natural extension of the radiation condition for the Schrödinger operators ([7], [11]). [10] also gave another proof of the limiting absorption principle for $H$.

In this work we are going to show the limiting absorption principle for $H$ whose separating surface $S$ satisfies a new condition (see Assumption 2.1) so that we can treat, for example, the case where $\Omega_{1}$ is an infinite cylindrical domain. Our proof of the limiting absorption principle will show that not only the uniqueness of the solution but also the existence of the limit (1.10) can be proved through the estimate of the radiation condition term

$$
\mathcal{D} u=\nabla u+\{(N-1) /(2 r)\} \widetilde{x} u-i k \widetilde{x} u,
$$

where

$$
\left\{\begin{array}{l}
u=R(z) f \\
f \in X \\
k=k(x, z)=\sqrt{z \mu(x)} \\
r=|x| \\
\widetilde{x}=x /|x|
\end{array}\right.
$$


The method demonstrated here can be applied to some other cases where the number of the media is more than 2 or infinitely many (multimedia cases). We shall discuss these cases with its short-range or long-range perturbation eleswhere $([9])$.

Another multimedia problem which has been discussed extensively is the stratified media in which the coefficients of the operator are the functions of $x^{\prime} \in \mathbf{R}^{k} \subset \mathbf{R}^{N}, k<N$. Some pertubed operators of the above type have been discussed, too. Here we refer Wilcox [16], Ben-Artzi-Dermanjian-Guillot [2], Weder [14], [15], DeBiévre-Pravica [4], [5], Boutet de Monvel-Berthier-Manda [3], and Zhang [17]. In [5] S. DeBiévre and D. W. Pravica proved that there is no point spectrum for the stratified propagators without any additional conditions other than sufficient smoothness of the coefficients at infinity. This is an extention of R. Weder [14]. In this work and also in the work [6], [13], and [10], we are interested in the non stratified case, in which it seems that the absence of the point spectrum can not be obtained without imposing some additional conditions.

In $\S 2$ we introduce the conditions on the separating surface $S$ and the function $\mu(x)$. In $\S 3 \sim \S 6$ we assume that $N \geq 3$. The uniqueness of the solution of the equation (1.12) with

$$
\liminf _{R \rightarrow \infty} \int_{S_{R}}|\mathcal{D} u|^{2} d S=0
$$

where $S_{R}$ is the sphere with radius $R$ and center at the origin, will be shown in $\S 3$. Our starting point in $\S 3$ is an identity involving the radiation condition term $\mathcal{D} u$ (Proposition 3.3). This is an extension of a similar identity in the case of Schrödinger operator ([7], [12]). Proposition 3.3 is also used in $\S 4$, where an estimate for the radiation condition term $\mathcal{D} u$ is given. In $§ 5$ some more estimates for $u=R(z) f$ will be given, and these estimates are combined in $\S 6$ to give the proof of the limiting absorption principle for $H$. We discuss the case that $N=2$ in $\S 7$ since we treat this case in a slightly different way although the result is rather similar to the case of $N \geq 3$. As for some technichal details of the computations and arguments appeared In $\S 3 \sim \S 7$, we refer to Jäger-Saitō [8].

Acknowledgement. This work was finished when the second author was visiting the University of Heidelberg from October 1994 through March 1995. Here he would like to thank Deutsche Forschungs Gemeinschaft for its support through SFB 359. Also the second author is thankful to Professor Willi Jäger for his kind hospitality during this period. 


\section{§2. The operator $H=-\mu(x)^{-1} \Delta$.}

We shall start with describing the conditions imposed on our operator.

Assumption 2.1. (i) Let $N$ be a positive integer such that $N \geq 2$ and let $\Omega_{\ell}, \ell=1,2$, are open sets of $\mathbf{R}^{N}$ satisfying (1.3).

(ii) Let the separating surface $S$ be defined by (1.6). The separating surface $S$ is assumed to be an $N$-1-dimensional continuous surface which consists of a finite number of smooth surfaces.

(iii) Let $\mu(x)$ is a positive function on $\mathbf{R}^{N}$ given by

$$
\mu(x)= \begin{cases}\mu_{1} & \left(x \in \Omega_{1}\right), \\ \mu_{2} & \left(x \in \Omega_{2}\right),\end{cases}
$$

where $\mu_{1}, \mu_{2}>0, \mu_{1} \neq \mu_{2}$. Further, we assume that

$$
\left(\mu_{2}-\mu_{1}\right)\left(x \cdot n^{(1)}\right)=\left(\mu_{1}-\mu_{2}\right)\left(x \cdot n^{(2)}\right) \geq 0
$$

for almost all $x \in S$, where $n^{(\ell)}, \ell=1,2$, is the outward unit normal of $\partial \Omega_{\ell}$ at $x$, and $x \cdot n^{(\ell)}$ is the inner product of $x$ and $n^{(\ell)}$ in $\mathbf{R}^{N}$.

Remark 2.2. The condition (2.2) requires that the inner products $x$. $n^{(1)}$ and $x \cdot n^{(2)}$ do not change their signs almost always on $S$. Note that the above assumption is satisfied if $\Omega_{1}$ is a cylindrical domain, $\mu_{1}<\mu_{2}$, and the origin is put in $\Omega_{1}$. Also (2.2) is satisfied when $S$ is an $(N-1$-dimensional) plane.

Definition 2.3. Let $X$ be the Hilbert space defined by

$$
X=L_{2}\left(\mathbf{R}^{N} ; \mu(x) d x\right)
$$

with its inner product $(,)_{X}$ and \|\|$_{X}$ given by

$$
\left\{\begin{array}{l}
(f, g)_{X}=\int_{\mathbf{R}^{N}} f(x) \overline{g(x)} \mu(x) d x \\
\|f\|_{X}=\left[(f, f)_{X}\right]^{1 / 2}
\end{array}\right.
$$

Then the operator $H$ in $X$ is defined by (1.4), that is,

$$
\left\{\begin{array}{l}
D(H)=H^{2}\left(\mathbf{R}^{N}\right), \\
H u=h u
\end{array}\right.
$$


where $h$ is given by (1.1). It is easy to see that $H$ is a selfadjoint operator in $X$.

\section{$\S 3$. The uniqueness of the solution.}

In this and the following three sections we assume that $N \geq 3$.

In order to discuss the uniqueness of the solution of the inhomogeneous equation

$$
-\mu(x)^{-1} \Delta u-\lambda u=f \quad(\lambda>0)
$$

with radiation condition, we shall start with some notations.

Notation 3.1. Let $z \in \mathbf{C}, x=\left(x_{1}, x_{2}, \cdots, x_{N}\right), r=|x|, \widetilde{x}=\left(\widetilde{x}_{1}, \widetilde{x}_{2}, \cdots\right.$, $\left.\widetilde{x}_{N}\right)=x / r, \partial_{j}=\partial / \partial x_{j}$ and $\nabla=\left(\partial / \partial x_{1}, \partial / \partial x_{2}, \cdots, \partial / \partial x_{N}\right)$. Then we set

(1) $k=k(x)=k(x, z)=[z \mu(x)]^{1 / 2}$, where the branch is taken so that $\operatorname{Im} k(x, z) \geq 0$

(2) $a=a(x)=a(x, z)=\operatorname{Re} k(x, z)$;

(3) $b=b(x)=b(x, z)=\operatorname{Im} k(x, z)$;

(4) $\mathcal{D}_{j} u=\partial_{j} u+\{(N-1) /(2 r)\} \widetilde{x}_{j} u-i k(x) \widetilde{x}_{j} u$, where $j=1,2, \cdots, N$;

(5) $\mathcal{D} u=\nabla u+\{(N-1) /(2 r)\} \widetilde{x} u-i k(x) \widetilde{x} u$;

(6) $\mathcal{D}_{r} u=\mathcal{D} u \cdot \widetilde{x}=\partial u / \partial r+\{(N-1) /(2 r)\} u-i k(x) u$;

(7) $\mathcal{D}_{n} u=\mathcal{D} u \cdot n=\partial u / \partial n+\{(N-1) /(2 r)\}(\widetilde{x} \cdot n) u-i k(x)(\widetilde{x} \cdot n) u$, where $n$ is a unit vector in $\mathbf{R}^{N}$.

Let $u \in H^{2}\left(\mathbf{R}^{N}\right)_{\text {loc }}$. Then the restrictions $\left.u\right|_{G}$ and $\left.\partial_{j} u\right|_{G}, j=1,2, \cdots, N$, of $u$ and $\partial_{j} u=\partial u / \partial x_{j}$ onto a smooth surface $G$ are defined as the traces of $u$ and $\partial_{j} u$ on $G$, respectively. Thus $\left.u\right|_{G}$ and $\left.\partial_{j} u\right|_{G}$ are considered to belong to $L_{2}(G)_{\text {loc }}$.

In this section we are going to prove the following theorem:

Theorem 3.2. Suppose that Assumption 2.1 with $N \geq 3$ holds. Let $u \in H^{2}\left(\mathbf{R}^{N}\right)_{\text {loc }}$ be a solution of the homogeneous equation

$$
-\mu(x)^{-1} \Delta u-\lambda u=0 \quad(\lambda>0)
$$

on $\mathbf{R}^{N}$ such that

$$
\liminf _{R \rightarrow \infty} \int_{S_{R}}\left(\left|\frac{\partial u}{\partial r}\right|^{2}+|u|^{2}\right) d S=0
$$


where

$$
S_{R}=\left\{x \in \mathbf{R}^{N}:|x|=R\right\} .
$$

Then $u$ is identically zero.

The proof will be divided into several steps. First, we are going to show an identity which directly follows from the equation $-\mu^{-1} \Delta u-z u=f$.

Proposition 3.3 (cf. [7], Lemma 2.2 and [12], Lemma 2.5.) Set

$$
f=\mu(x)^{-1}\left(-\Delta u-k^{2} u\right),
$$

where $u \in H^{2}\left(\mathbf{R}^{N}\right)_{\text {loc }}$. Let $\xi$ be a real-valued, continuous function on $[0, \infty)$ such that $\xi$ has piecewise continuous derivative. Set $\varphi(x)=\alpha(x) \xi(|x|)$, where $\alpha$ is a simple function which is constant on each $\Omega_{\ell}$. For $0<r<R<\infty$, set

$$
B_{r R}=\left\{x \in \mathbf{R}^{N}: r<|x|<R\right\},
$$

Then we have

$$
\begin{array}{rl}
\int_{B_{r R}}\left(b \varphi+\frac{1}{2} \frac{\partial \varphi}{\partial r}\right)|\mathcal{D} u|^{2} & d x+\sum_{\ell=1}^{2} \int_{\partial \Omega_{\ell \cap B_{r R}}} \varphi \operatorname{Im}\left\{\bar{k} \frac{\partial u}{\partial n} \bar{u}\right\} d S \\
& +\int_{B_{r R}}\left(\frac{\varphi}{r}-\frac{\partial \varphi}{\partial r}\right)\left(|\mathcal{D} u|^{2}-|\mathcal{D} r u|^{2}\right) d x \\
& +c_{N} \int_{B_{r R}} r^{-2}\left(\frac{\varphi}{r}-2^{-1} \frac{\partial \varphi}{\partial r}+b \varphi\right)|u|^{2} d x \\
= & \operatorname{Re} \int_{B_{r R}} \varphi \mu(x) f \overline{\mathcal{D}_{r} u} d x \\
+ & 2^{-1} \sum_{\ell=1}^{2} \int_{\partial \Omega_{\ell} \cap B_{r R}} \varphi\left\{\frac{(N-1) b}{r}+|k|^{2}\right\}(\widetilde{x} \cdot n)|u|^{2} d S \\
+ & 2^{-1} \int_{S_{R}}^{\varphi} \varphi\left(2\left|\mathcal{D}_{r} u\right|^{2}-|\mathcal{D} u|^{2}-c_{N} r^{-2}|u|^{2}\right) d S \\
- & 2^{-1} \int_{S_{r}}^{\varphi} \varphi\left(2\left|\mathcal{D}_{r} u\right|^{2}-|\mathcal{D} u|^{2}-c_{N} r^{-2}|u|^{2}\right) d S,
\end{array}
$$

where $\Omega_{1}, \Omega_{2}$ satisfies (1.3), $S$ is as in (ii) of Assumption 2.1, $\partial / \partial n$ in the integrand of the surface integral over $\partial \Omega_{\ell} \cap B_{r R}$ means the directional derivative in the direction of the outward normal $n=n^{(\ell)}$ of $\partial \Omega_{\ell}$, and

$$
c_{N}=(N-1)(N-3) / 4 \text {. }
$$

To prove Proposition 3.3 we first rewrite (3.5) as

$$
-\sum_{j=1}^{N} \partial_{j} \mathcal{D}_{j} u+\left\{\frac{N-1}{2 r}-i k\right\} \mathcal{D}_{r} u+\frac{c_{N}}{r^{2}} u=\mu(x) f
$$


Then (3.7) is obtained by multiplying bothe sides of (3.9) by $\varphi \overline{\mathcal{D}_{r} u}$, taking the real part and using partial integration. For the details of computation see Appendix, A.1 of [8]. The following lemmas are also used for the proof of Theorem 3.2.

Lemma 3.4. Let $\varphi(x)=\xi(|x|)$ and let $\xi$ be a continuous function on $[0, \infty)$ such that $\xi$ has piecewise bounded continuous derivative $\xi^{\prime}$ and $\xi(0)=$ 0 . Let $S$ be an $N-1$-dimensional continuous surface which consists of a finite number of smooth surfaces. Let $F(x)$ be a locally $L_{1}$ function with locally $L_{1}$ derivatives in a neighborhood of $S$. Then we have, $R>0$,

$$
\int_{B_{R} \cap S} \varphi(|x|) F(x) d S=\int_{0}^{R} \frac{\partial \varphi}{\partial r}\left(\int_{B_{r R} \cap S} F(x) d S\right) d r
$$

where, for $0<r<R<\infty, B_{r R}$ is as in (3.6), and $B_{R}=\left\{x \in \mathbf{R}^{N}:|x|<\right.$ $R\}$ is an open ball with origin 0 and radius $R$.

Proof. Since $F(x)$ can be approximated by a sequence of $C^{1}$ functions in a neighborhood of $S$, we may assume that $F$ is a $C^{1}$ function. For $\epsilon>0$ set

$$
S_{\epsilon}=\left\{x \in \mathbf{R}^{N}: \operatorname{dist}(x, S)<\epsilon\right\}
$$

where $\operatorname{dist}(x, S)$ is the distance between $x$ and $S$, and let $\chi_{S, \epsilon}(x)$ be the characteristic function of the set $S_{\epsilon}$. Then we have by an easy computation

$$
\begin{aligned}
\int_{0}^{R} \frac{\partial \varphi}{\partial r}\left(\int_{B_{r R} \cap S_{\epsilon}}\right. & F(x) d x) d r \\
& =\int_{0}^{R} \xi^{\prime}(r)\left(\int_{B_{r R}} \chi_{S, \epsilon}(x) F(x) d S\right) d r \\
& =\int_{0}^{R} \xi^{\prime}(r) \int_{r}^{R}\left(\int_{S_{t}} \chi_{S, \epsilon}(x) F(x) d S\right) d t d r \\
& =\int_{0}^{R} \int_{0}^{t} \xi^{\prime}(r)\left(\int_{S_{t}} \chi_{S, \epsilon}(x) F(x) d S\right) d r d t \\
& =\int_{0}^{R} \xi(t)\left(\int_{S_{t}} \chi_{S, \epsilon}(x) F(x) d S\right) d t \\
& =\int_{B_{R}}^{\varphi(|x|) \chi_{S, \epsilon}(x) F(x) d x}
\end{aligned}
$$

The equality (3.10) (for smooth $F$ ) is obtained by dividing both sides of (3.12) by $\epsilon$ and letting $\epsilon \downarrow 0$, which completes the proof. \|

Lemma 3.5. Let $u \in H^{2}\left(\mathbf{R}^{N}\right)_{\text {loc }}$ be a solution of the homogeneous equation (3.2) with $\lambda>0$. Let $\Omega_{1}$ and $\Omega_{2}$ satisfy (i) and (ii) of Assumption 2.1 . 
(i) Let $0<r<R<\infty$. Then we have

$$
\begin{aligned}
\sum_{\ell=1}^{2} \int_{\partial \Omega_{\ell} \cap B_{r R}} \operatorname{Im} & \left\{k \frac{\partial u}{\partial n} \bar{u}\right\} d S \\
& =-\int_{S_{R}} \operatorname{Im}\left\{k \frac{\partial u}{\partial r} \bar{u}\right\} d S+\int_{S_{r}} \operatorname{Im}\left\{k \frac{\partial u}{\partial r} \bar{u}\right\} d S
\end{aligned}
$$

where $B_{r R}$ is given by (3.6).

(ii) Let $\varphi(x)=\xi(|x|)$ and let $\xi$ be a real-valued, continuous function on $[0, \infty)$ such that $\xi$ has piecewise continuous derivative $\xi^{\prime} \geq 0$, and $\xi(0)=0$. Then we have, for $0<R<\infty$,

$$
\begin{gathered}
\int_{B_{R}} \frac{1}{2} \frac{\partial \varphi}{\partial r}|\mathcal{D} u|^{2} d x+\sum_{\ell=1}^{2} \int_{\partial \Omega_{\ell} \cap B_{R}} \varphi \operatorname{Im}\left\{k \frac{\partial u}{\partial n} \bar{u}\right\} d S \\
\geq \int_{B_{R}} \frac{1}{2} \frac{\partial \varphi}{\partial r} k^{2}|u|^{2} d x+\int_{B_{R}} \frac{1}{2} \frac{\partial \varphi}{\partial r}\left(|\nabla u|^{2}-\left|\frac{\partial u}{\partial r}\right|^{2}\right) d x \\
-\xi(R) \int_{S_{R}} \operatorname{Im}\left\{k \frac{\partial u}{\partial r} \bar{u}\right\} d S
\end{gathered}
$$

where $k=k(x)=\sqrt{\lambda \mu(x)}$.

Proof. (I) Multiply both side of

$$
-\Delta u-\mu(x) \lambda u=0
$$

by $k \bar{u}$ and integrate over $B_{r R}$. Then, (3.13) is obtained by taking the imaginary part and using partial integration.

(II) It follows from (3.13) and Lemma 3.4 that

$$
\begin{aligned}
& \sum_{\ell=1}^{2} \int_{\partial \Omega_{\ell} \cap B_{R}} \varphi \operatorname{Im}\left\{k \frac{\partial u}{\partial n} \bar{u}\right\} d S \\
& =\sum_{\ell=1}^{2} \int_{0}^{R} \frac{\partial \varphi}{\partial r}\left(\int_{\partial \Omega_{\ell} \cap B_{r R}} \operatorname{Im}\left\{k \frac{\partial u}{\partial n} \bar{u}\right\} d S\right) d r \\
& =-\int_{0}^{R} \frac{\partial \varphi}{\partial r}\left(\int_{S_{R}} \operatorname{Im}\left\{k \frac{\partial u}{\partial r} \bar{u}\right\} d S\right) d r \\
& \quad+\int_{0}^{R} \frac{\partial \varphi}{\partial r}\left(\int_{S_{r}} \operatorname{Im}\left\{k \frac{\partial u}{\partial r} \bar{u}\right\} d S\right) d r
\end{aligned}
$$




$$
=-\xi(R) \int_{S_{R}} \operatorname{Im}\left\{k \frac{\partial u}{\partial r} \bar{u}\right\} d S+\int_{B_{R}} \frac{\partial \varphi}{\partial r} \operatorname{Im}\left\{k \frac{\partial u}{\partial r} \bar{u}\right\} d x .
$$

(III) Now we are going to evaluate the term $|\mathcal{D} u|^{2}$. By definition and the Schwarz inequality it follows that

$$
\begin{aligned}
&|\mathcal{D} u|^{2}=|\nabla u|^{2}-2 \operatorname{Im}\left(k \frac{\partial u}{\partial r} \bar{u}\right)+k^{2}|u|^{2} \\
&+\frac{N-1}{r} \operatorname{Re}\left(\frac{\partial u}{\partial r} \bar{u}\right)+\frac{(N-1)^{2}}{4 r^{2}}|u|^{2} . \\
& \geq-2 \operatorname{Im}\left(k \frac{\partial u}{\partial r} \bar{u}\right)+\left(|\nabla u|^{2}-\left|\frac{\partial u}{\partial r}\right|^{2}\right)+k^{2}|u|^{2} .
\end{aligned}
$$

Multiply both side of (3.17) by $\partial \varphi / \partial r$ and integrate over $B_{R}$. Then, using (3.16), too, we have (3.14). $\quad$ |

Now we are in a position to prove Theorem 3.2. Here and in the sequel we agree that $C=C(A, B, \cdots)$ in an inequality means a positive constant depending on $A, B, \cdots$. But very often symbols indicating obvious dependence such as the operator $H$ will be left out.

Proof of Theorem 3.2. Let $u \in H^{2}\left(\mathbf{R}^{N}\right)_{\text {loc }}$ be a solution of the homogeneous equation (3.2). Let $R_{0} \geq 1$ and define $\varphi$ by

$$
\varphi(x)= \begin{cases}|x| & \left(0 \leq|x| \leq R_{0}\right) \\ R_{0} & \left(|x|>R_{0}\right) .\end{cases}
$$

The function $\varphi$ satisfies the conditions given in Proposition 3.3 and Lemma 3.5. Then it follows from (3.7) in Proposition 3.3 with $f=0, b=0, k=$ $\sqrt{\lambda \mu(x)}$ and Lemma 3.5, (ii) that, for any $R>R_{0}>r>0$,

$$
\begin{aligned}
\int_{B_{r R_{0}}} & \frac{1}{2} k^{2}|u|^{2} d x \\
\leq & R_{0} C \int_{S_{R}}\left(\left|\frac{\partial u}{\partial r}\right|^{2}+|u|^{2}\right) d S \\
& +2^{-1} \int_{S_{r}} r\left(|\mathcal{D} u|^{2}+c_{N} r^{-2}|u|^{2}\right) d S \\
& +\int_{B_{r}} \frac{1}{2} r|\mathcal{D} u|^{2} d x+\sum_{\ell=1}^{2} \int_{\partial \Omega_{\ell} \cap B_{r}} r \operatorname{Im}\left\{k \frac{\partial u}{\partial n} \bar{u}\right\} d S
\end{aligned}
$$


with a positive constant $C=C(\lambda)$, where we have used the facts that

$$
\left\{\begin{array}{l}
\frac{\varphi}{r}-2^{-1} \frac{1}{2} \frac{\partial \varphi}{\partial r} \geq 0 \\
|\nabla u|^{2}-\left|\frac{\partial u}{\partial r}\right|^{2} \geq 0 \\
2\left|\mathcal{D}_{r} u\right|^{2}-|\mathcal{D} u|^{2}-c_{N} r^{-2}|u|^{2} \\
\quad=\left|\mathcal{D}_{r} u\right|^{2}-\left(|\mathcal{D} u|^{2}-\left|\mathcal{D}_{r} u\right|^{2}\right)-c_{N} r^{-2}|u|^{2} \leq\left|\mathcal{D}_{r} u\right|^{2} \\
-2\left|\mathcal{D}_{r} u\right|^{2}+|\mathcal{D} u|^{2}+c_{N} r^{-2}|u|^{2} \leq|\mathcal{D} u|^{2}+c_{N} r^{-2}|u|^{2} \\
\sum_{\ell=1}^{2} \int_{\partial \Omega_{\ell} \cap B_{R}} \varphi|k|^{2}(\widetilde{x} \cdot n)|u|^{2} d S \\
=\lambda \int_{S \cap B_{R}} \varphi\left(\mu_{1}-\mu_{2}\right)\left(\widetilde{x} \cdot n^{(1)}\right)|u|^{2} d S \leq 0
\end{array}\right.
$$

Here the last inequality follows from (2.2) in Assumption 2.1. Since $u \in$ $H^{2}\left(\mathbf{R}^{N}\right)_{\text {loc }}$ with $N \geq 3$, it follows from the Hardy inequality that $u / r$ is locally $L_{2}\left(\mathbf{R}^{N}\right)$, and hence the second term of the right-hand side of (3.19) tends to zero as $r \rightarrow 0$ along a suitable sequence $\left\{r_{m}\right\}$, i.e.,

$$
\int_{S_{r_{m}}} r\left(|\mathcal{D} u|^{2}+c_{N} r^{-2}|u|^{2}\right) d S \rightarrow 0
$$

as $r_{m} \rightarrow 0$. The last two terms of the right-hand side of (3.19) tend to 0 as $r \downarrow 0$, since their integrands are integrable. Thus we have

$$
\int_{B_{R_{0}}} \frac{1}{2} k^{2}|u|^{2} d x \leq R_{0} C \int_{S_{R}}\left(\left|\frac{\partial u}{\partial r}\right|^{2}+|u|^{2}\right) d S \quad\left(R>R_{0}\right)
$$

Therefore, by letting $R \rightarrow \infty$ along an appropriate sequence $\left\{R_{m}\right\}$, the righthand side of (3.22) becomes 0, i.e., we have,

$$
\int_{B_{R_{0}}} \frac{1}{2} k^{2}|u|^{2} d x=0
$$

for any $R_{0} \geq 1$, which implies that $u$ is identically zero. $\|$

Using Theorem 3.2, we can easily show the nonexistence of the eigenvalues of the operator $H$.

Corollary 3.6. Suppose Assumption 2.1 with $N \geq 3$ holds. Then the operator $H$ has no eigenvalues. 
Proof. Since $H$ is nonnegative, we have only to show that $H$ has no nonnegative eigenvalues. Suppose that $u \in D(H)=H^{2}\left(\mathbf{R}^{N}\right)$ be an eigenfunction associated with a positive eigenvalue $\lambda$ of $H$. Then, since $u$ is a solution of (3.2) and satisfies the condition (3.3), $u$ is identically zero, which is a contradiction. Suppose that 0 is an eigenvalue of $H$ and $u$ is the corresponding eigenfunction. Then $u$ becomes also an eigenfunction of the operator $-\Delta$ and $\lambda=0$ becomes an eigenvalue of $-\Delta$, which is again a contradiction since $-\Delta$ does not have eigenvalue $\lambda=0$. $\|$

Finally we shall show that the radiation condition

$$
\liminf _{R \rightarrow \infty} \int_{S_{R}}\left|\mathcal{D}_{r}^{( \pm)} u\right|^{2} d S=0
$$

or

$$
\liminf _{R \rightarrow \infty} \int_{S_{R}}\left|\frac{\partial u}{\partial r} \mp i k u\right|^{2} d S=0
$$

implies that (3.3) for a solution $u \in H^{2}\left(\mathbf{R}^{N}\right)_{\text {loc }}$ of the equation (3.2). Here $k=\sqrt{\lambda \mu(x)}$ and $\mathcal{D}_{r}^{( \pm)} u$ is given by

$$
\mathcal{D}_{r}^{( \pm)} u=\partial u / \partial r+\{(N-1) /(2 r)\} u \mp i k(x) u
$$

Theorem 3.7. Suppose Assumption 2.1 with $N \geq 3$ holds. Let $u \in$ $H^{2}\left(\mathbf{R}^{N}\right)_{\text {loc }}$ be a solution of the homogeneous equation (3.2) with $\lambda>0$. Suppose that (3.24) or (3.25) holds. Then $u$ is identically zero.

Proof. We have only to show that the condition (3.24) or (3.25) implies (3.3). Here we shall consider the condition

$$
\liminf _{R \rightarrow \infty} \int_{S_{R}}\left|\mathcal{D}_{r}^{(+)} u\right|^{2} d S=0
$$

All the other conditions can be treated similarly. Multiply both sides of (3.2) by $\bar{u}$, integrate over $B_{R}$ with $R>0$ and take the imaginary part. Then we obtain

$$
\operatorname{Im} \int_{S_{R}} \frac{\partial u}{\partial r} \bar{u} d S=0
$$

Since we have from (3.28)

$$
\operatorname{Im} \int_{S_{R}}\left(\mathcal{D}_{r}^{(+)} u\right) \bar{u} d S=-\int_{S_{R}} k|u|^{2} d S
$$


with $k=\sqrt{\lambda \mu}$, it follows that

$$
\begin{aligned}
k_{0} \int_{S_{R}}|u|^{2} d S & \leq \int_{S_{R}}\left|\mathcal{D}_{r}^{(+)} u\right||u| d S \\
& \leq \frac{k_{0}}{2} \int_{S_{R}}|u|^{2} d S+\frac{1}{2 k_{0}} \int_{S_{R}}\left|\mathcal{D}_{r}^{(+)} u\right|^{2} d S
\end{aligned}
$$

or

$$
\int_{S_{R}}|u|^{2} d S \leq \frac{1}{k_{0}^{2}} \int_{S_{R}}\left|\mathcal{D}_{r}^{(+)} u\right|^{2} d S
$$

where $k_{0}=\sqrt{\lambda \min \left(\mu_{1}, \mu_{2}\right)}$. On the other hand we have

$$
\int_{S_{R}}\left|\frac{\partial u}{\partial r}\right|^{2} d S \leq 2 \int_{S_{R}}\left|\mathcal{D}_{r}^{(+)} u\right|^{2} d S+2\left(\left(\frac{N-1}{2}\right)^{2}+k_{1}^{2}\right) \int_{S_{R}}|u|^{2} d S
$$

for $R \geq 1$ with $k_{1}=\sqrt{\lambda \max \left(\mu_{1}, \mu_{2}\right)}$. Thus it follows from (3.31) and (3.32) that there exists a positive constants $C=C(\lambda)$ such that

$$
\int_{S_{R}}\left(\left|\frac{\partial u}{\partial r}\right|^{2}+|u|^{2}\right) d S \leq C \int_{S_{R}}\left|\mathcal{D}_{r}^{(+)} u\right|^{2} d S
$$

which completes the proof. \|

Later we shall need the following corollary which guarantees the uniqueness of the inhomogenous equation

$$
-\mu(x)^{-1} \Delta u-\lambda u=f
$$

with one of the conditions

$$
\left\{\begin{array}{l}
\int_{E_{R}} \frac{1}{r}\left|\mathcal{D}_{r}^{( \pm)} u\right|^{2} d x<\infty \\
\int_{E_{R}} \frac{1}{r}\left|\frac{\partial u}{\partial r} \mp i k u\right|^{2}, d x<\infty
\end{array}\right.
$$

where $E_{R}=\left\{x \in \mathbf{R}^{N}:|x|>R\right\}, R>0$.

Corollary 3.8. Let $\lambda>0$ and let $f \in L_{2}\left(\mathbf{R}^{N}\right)_{\text {loc }}$. Then the solution $u \in H^{2}\left(\mathbf{R}^{N}\right)_{\text {loc }}$ of the equation (3.34) with one of the radiation conditions in (3.35) is unique.

Proof. Let $u_{1}$ and $u_{2}$ be the solutions of the equation (3.34) satisfying, say,

$$
\int_{E_{R}} \frac{1}{r}\left|\mathcal{D}_{r}^{(+)} u_{j}\right|^{2} d x<\infty \quad(j=1,2)
$$


with $R>0$. Set $u=u_{1}-u_{2}$.. Then $u$ is a solution of the homogeneous equation (3.2) and satisfies (3.36) with $u$ replaced by $u_{1}-u_{2}$, which implies that

$$
\liminf _{R \rightarrow \infty} \int_{S_{R}}\left|\mathcal{D}_{r}^{(+)} u\right|^{2} d S=0 .
$$

Therefore we have $u=0$ from Theorem 3.7. \|

\section{§4. An estimate for the radiation condition.}

Let $L_{2, t}\left(\mathbf{R}^{N}\right)$ be the weighted Hilbert space defined by (1.11). Let the resolvent $(H-z)^{-1}$ of the operator $H$ will be denoted by $R(z)$. Now consider $u \in X$ defined by

$$
\left\{\begin{array}{l}
u=R(z) f \\
z=\lambda+i \eta \\
f \in L_{2, \delta}\left(\mathbf{R}^{N}\right) .
\end{array} \quad(\lambda \geq 0, \eta \neq 0)\right.
$$

In this section we are going to prove the following

Theorem 4.1. Suppose that Assumption 2.1 holds with $N \geq 3$. Let $1 / 2<\delta \leq 1$. Let $u$ be given by (4.1). Then there exists a positive constant $C=C(\delta)$ such that

$$
\|\mathcal{D} u\|_{\delta-1} \leq C\|f\|_{\delta}
$$

where $\mathcal{D} u$ is as in Notation 3.1, \|\|$_{t}$ is the norm of $L_{2, t}\left(\mathbf{R}^{N}\right)$, and the constant $C(\delta)$ is independent of $f$ and $z$ satisfying (4.1).

In order to show the theorem we need a lemma.

Lemma 4.2. Let $z=\lambda+i \eta \in \mathbf{C} \backslash \mathbf{R}$. Let $a, b, k$ be as in Notation 3.1. Then we have

$$
\begin{cases}a=a(x, z)=\sqrt{\mu(x)} c_{a}(z) & \left(c_{a}(z)=\frac{\eta}{|\eta|} \sqrt{\frac{|z|+\lambda}{2}}\right) \\ b=b(x, z)=\sqrt{\mu(x)} c_{b}(z) & \left(c_{b}(z)=\frac{|\eta|}{\sqrt{2(|z|+\lambda)}}\right) \\ |k|^{2}=|k(x, z)|^{2}=\mu(x)|z| . & \end{cases}
$$

Since the lemma is shown by an easy computation, we shall omit the proof. 
Proof of Theorem 4.1. (I) Let $\xi(r)$ be defined by

$$
\xi(r)= \begin{cases}r & (0 \leq r \leq 1), \\ 2^{-(2 \delta-1)}(1+r)^{2 \delta-1} & (r \geq 1)\end{cases}
$$

Set $\varphi(x)=\xi(r) / \sqrt{\mu(x)}$, where $r=|x|$, and $u=R(z) f$ in (3.7) of Proposition 3.3. We are going to evaluate each term of the left-hand side and the right-hand side of (3.7). Here we set $0<r<1<R$.

(II) Let

$$
I_{L 1}=\int_{B_{r R}}\left(b \varphi+\frac{1}{2} \frac{\partial \varphi}{\partial r}\right)|\mathcal{D} u|^{2} d x
$$

Then we have

$$
\begin{aligned}
I_{L 1} \geq \frac{1}{2} \int_{B_{r 1}} \frac{1}{\sqrt{\mu(x)}}|\mathcal{D} u|^{2} d x & \\
& \quad+\frac{1}{2} \int_{B_{1 R}} \frac{2 \delta-1}{2^{2 \delta-1} \sqrt{\mu(x)}}(1+r)^{2 \delta-2}|\mathcal{D} u|^{2} d x \\
\geq & \frac{c_{\delta}}{2 \sqrt{M_{0}}} \int_{B_{r R}}(1+r)^{2 \delta-2}|\mathcal{D} u|^{2} d x,
\end{aligned}
$$

where $M_{0}=\max \left(\mu_{1}, \mu_{2}\right)$, and

$$
c_{\delta}=\frac{2 \delta-1}{2^{2 \delta-1}}
$$

(III) Let the second term of the left-hand side of (3.7) be denoted by $I_{L 2}$. Note that we have

$$
\begin{aligned}
\int_{\partial \Omega_{\ell} \cap B_{r R}} \varphi & (x) \operatorname{Im}\left\{\bar{k} \frac{\partial u}{\partial n} \bar{u}\right\} d S \\
= & \int_{S \cap B_{r R}} \frac{\xi(r)}{\sqrt{\mu_{\ell}}} \sqrt{\mu_{\ell}} \operatorname{Im}\left\{c_{-}(z) \frac{\partial u}{\partial n^{(\ell)}} \bar{u}\right\} d S \\
= & \int_{S \cap B_{r R}} \xi(r) \operatorname{Im}\left\{c_{-}(z) \frac{\partial u}{\partial n^{(\ell)}} \bar{u}\right\} d S \quad(\ell=1,2),
\end{aligned}
$$

where $c_{-}(z)=c_{a}(z)-i c_{b}(z)$, and $c_{a}(z)$ and $c_{b}(z)$ are as in Lemma 4.2. Noting that $n^{(1)}+n^{(2)}=0$, we have $I_{L 2}=0$.

(IV) Set

$$
I_{L 3}=\int_{B_{r R}}\left(\frac{\varphi}{r}-\frac{\partial \varphi}{\partial r}\right)\left(|\mathcal{D} u|^{2}-\left|\mathcal{D}_{r} u\right|^{2}\right) d x
$$


Since it is easy to see that the integrand is nonnegative, we have $I_{L 3} \geq 0$. Similarly we have

$$
I_{L 4}=c_{N} \int_{B_{r R}} r^{-2}\left(\frac{\varphi}{r}-2^{-1} \frac{\partial \varphi}{\partial r}+b \varphi\right)|u|^{2} d x \geq 0 .
$$

(V) Using the Schwarz inequality, we have

$$
\begin{aligned}
I_{R 1}= & \operatorname{Re} \int_{B_{r R}} \varphi \mu(x) f \overline{\mathcal{D}_{r} u} d x \\
& \leq \sqrt{M_{0}} \int_{B_{r R}}(1+r)^{2 \delta-1}|f| \mathcal{D}_{r} u \mid d x \\
& \leq \frac{M_{0}}{4 \epsilon} \int_{B_{r R}}(1+r)^{2 \delta}|f|^{2} d x+\epsilon \int_{B_{r R}}(1+r)^{2 \delta-2}|\mathcal{D} u|^{2} d x,
\end{aligned}
$$

where $\epsilon$ is an arbitrary positive number.

(VI) Let $I_{R 2}$ be the second term of the right-hand side of (3.7). Then,

$$
\begin{aligned}
I_{R 2}= & 2^{-1}\left(\sum_{\ell=1}^{2} \int_{\partial \Omega_{\ell} \cap B_{r R}} \varphi \frac{(N-1) b}{r}(\widetilde{x} \cdot n)|u|^{2} d S\right. \\
& \left.+\sum_{\ell=1}^{2} \int_{\partial \Omega_{\ell} \cap B_{r R}} \varphi|k|^{2}(\widetilde{x} \cdot n)|u|^{2} d S\right) \\
\equiv & 2^{-1}\left[I_{R 2}^{(1)}+I_{R 2}^{(2)}\right] .
\end{aligned}
$$

Here, as in (II), we see from Lemma 4.2 that $I_{R 2}^{(1)}=0$ and

$$
\begin{aligned}
I_{R 2}^{(2)}= & \int_{S \cap B_{r R}} \frac{\xi(r)}{\sqrt{\mu_{1}}} \mu_{1}|z|\left(\widetilde{x} \cdot n^{(1)}\right)|u|^{2} d S \\
& +\int_{S} \frac{\xi(r)}{\sqrt{\mu_{2}}} \mu_{2}|z|\left(\widetilde{x} \cdot n^{(2)}\right)|u|^{2} d S \\
= & \int_{S \cap B_{r R}} \xi(r)\left(\sqrt{\mu_{1}}-\sqrt{\mu_{2}}\right)|z|\left(\widetilde{x} \cdot n^{(1)}\right)|u|^{2} d S \\
= & \int_{S \cap B_{r R}} \frac{\xi(r)}{\sqrt{\mu_{1}}+\sqrt{\mu_{2}}}\left(\mu_{1}-\mu_{2}\right)|z|\left(\widetilde{x} \cdot n^{(1)}\right)|u|^{2} d S \\
\leq & 0,
\end{aligned}
$$

where we have used (2.2) in Assumption 2.1 again. Thus we have $I_{R 2} \leq 0$.

(VII) It follows from (II) (VI) that 


$$
\begin{aligned}
& \frac{c_{\delta}}{4 \sqrt{M_{0}}} \int_{B_{r R}}(1+r)^{2 \delta-2}|\mathcal{D} u|^{2} d x \\
& \leq \frac{M_{0}^{3 / 2}}{c_{\delta}} \int_{B_{r R}}(1+r)^{2 \delta}|f|^{2} d x \\
& +2^{-1} \int_{S_{R}} \varphi\left(\left|\mathcal{D}_{r} u\right|^{2} d S\right. \\
& \quad+2^{-1} \int_{S_{r}} \varphi\left(|\mathcal{D} u|^{2}+c_{N} r^{-2}|u|^{2}\right) d S
\end{aligned}
$$

where we set

$$
\epsilon=\frac{c_{\delta}}{4 \sqrt{M_{0}}}
$$

in (4.11), and we have used the third and fourth inequalities in (3.20) to eveluate the third and fourth term of the right-hand side of (3.7), Proceeding as in the proof of Theorem 3.2, we can let $R \uparrow \infty$ and $r \downarrow 0$ along suitable sequences $\left\{R_{n}\right\}$ and $\left\{r_{n}\right\}$ to obtain

$$
\begin{aligned}
\frac{c_{\delta}}{2 \sqrt{M_{0}}} \int_{\mathbf{R}^{N}} & (1+r)^{2 \delta-2}|\mathcal{D} u|^{2} d x \\
& \leq \frac{M_{0}^{3 / 2}}{c_{\delta}} \int_{\mathbf{R}^{N}}(1+r)^{2 \delta}|f|^{2} d x,
\end{aligned}
$$

which completes the proof. \|

Theorem 4.1 is combined with the inequality

$$
|\nabla u-i k \widetilde{x} u|^{2} \leq 2|\mathcal{D} u|^{2}+\frac{(N-1)^{2}}{2 r^{2}}|u|^{2}
$$

to obtain the following corollary:

Corollary 4.3. Suppose that Assumption 2.1 with $N \geq 3$ holds. Let $1 / 2<\delta \leq 1$. Let $u$ be given by (4.1). Then there exists a positive constant $C=C(\delta)$ such that

$$
\int_{E_{1}}(1+r)^{2 \delta-2}|\nabla u-i k \widetilde{x} u|^{2} d x \leq C\left(\|f\|_{\delta}^{2}+\|u\|_{-\delta}^{2}\right),
$$

where

$$
E_{1}=\left\{x \in \mathbf{R}^{N}:|x|>1\right\} .
$$




\section{$\S 5$. Boundedness of $R(z)$.}

Using the estimates for the radiation condition term $\mathcal{D} u(u=R(z) f)$, which were given in the preceding section, we are going to prove several uniform boundedness estimates for $R(z)$. At the same time the first theorems (Theorems 5.1 and 5.2) will prepare the arguments given in $\S 6$, where we shall discuss the limiting absorption principle for the operator $H$.

Theorem 5.1. Suppose that Assumption 2.1 holds with $N \geq 3$. Let $1 / 2<\delta \leq 1$. Let $u=R(z) f$, where $f \in L_{2, \delta}\left(\mathbf{R}^{N}\right)$ and $z=\lambda+i \eta$ with $\lambda \geq 0, \eta \neq 0$. Then there exists a positive constant $C=C(\delta)$ such that

$$
\begin{aligned}
\int_{E_{s}}(1+r)^{-2 \delta}\left(|\nabla u|^{2}+|k|^{2}|u|^{2}\right) d x & \\
& \leq C(1+\sqrt{|z|})(1+s)^{-(2 \delta-1)}\left(\|f\|_{\delta}^{2}+\|u\|_{-\delta}^{2}\right) \quad(s \geq 1),
\end{aligned}
$$

where $E_{s}=\left\{x \in \mathbf{R}^{N}:|x|>s\right\}$, and \|\|$_{t}$ is the norm of $L_{2, t}\left(\mathbf{R}^{N}\right)$. The constant $C=C(\delta)$ is independent of $f$ and $z$ satisfying the above conditions and $s \geq 1$.

Proof. Let $\alpha(x)=1 / \sqrt{\mu(x)}$. Then. as we have seen in (III) of the proof of Theorem 4.1, we have

$$
\sum_{\ell=1}^{2} \int_{\partial \Omega_{\ell} \cap B_{r}} \alpha \operatorname{Im}\left\{\bar{k} \frac{\partial u}{\partial n} \bar{u}\right\} d S=0 .
$$

Multiply both sides of the equation $\mu f=-\Delta u-k^{2} u$ by $\alpha \bar{k} \bar{u}$, integrate over $B_{r}$ and take the imaginary part to obtain

$$
\begin{gathered}
\int_{B_{r}} \alpha \mu \operatorname{Im}(\bar{k} f \bar{u}) d x=-\int_{B_{r}} b \alpha\left(|\nabla u|^{2}+|k|^{2}|u|^{2}\right) d x \\
-\int_{S_{r}} \alpha \operatorname{Im}\left(\bar{k} \frac{\partial u}{\partial r} \bar{u}\right) d S
\end{gathered}
$$

where we have used (5.2). Combining

$$
|\nabla u-i k \widetilde{x} u|^{2}=|\nabla u|^{2}+|k|^{2}|u|^{2}-2 \operatorname{Im}\left(\bar{k} \frac{\partial u}{\partial r} \bar{u}\right)
$$


with (5.3), we obtain

$$
\begin{aligned}
\int_{S_{r}} \alpha\left(|\nabla u|^{2}+|k|^{2}|u|^{2}\right) d S & \\
= & \int_{S_{r}} \alpha|\nabla u-i k \widetilde{x} u|^{2} d S-2 \int_{B_{r}} \alpha \mu \operatorname{Im}(\bar{k} f \bar{u}) d x \\
& \quad-\int_{B_{r}} b \alpha\left(|\nabla u|^{2}+|k|^{2}|u|^{2}\right) d x \\
\leq & \int_{S_{r}} \alpha|\nabla u-i k \widetilde{x} u|^{2} d S+2 \sqrt{M_{0}}\|f\|_{\delta}\|k u\|_{-\delta}
\end{aligned}
$$

Multiply both sides of $(5.5)$ by $(1+r)^{-2 \delta}$ and integrate from $s$ to $\infty$. Then, setting $\mu_{0}=\min \left(\mu_{1}, \mu_{2}\right)$ and $M_{0}=\max \left(\mu_{1}, \mu_{2}\right)$, we have

$$
\begin{aligned}
\frac{1}{\sqrt{M_{0}}} & \int_{E_{s}}(1+r)^{-2 \delta}\left(|\nabla u|^{2}+|k|^{2}|u|^{2}\right) d x \\
\leq & \frac{1}{\sqrt{\mu_{0}}} \int_{E_{s}}(1+r)^{-2 \delta}|\nabla u-i k \widetilde{x} u|^{2} d x \\
& \quad+\frac{2 \sqrt{M_{0}}}{(2 \delta-1)}(1+s)^{-(2 \delta-1)}\|f\|_{\delta}\|k u\|_{-\delta} \\
\leq & \frac{(1+s)^{-(4 \delta-2)}}{\sqrt{\mu_{0}}} \int_{E_{s}}(1+r)^{2 \delta-2}|\nabla u-i k \widetilde{x} u|^{2} d x \\
& +\frac{2 \sqrt{M_{0}}}{(2 \delta-1)}(1+s)^{-(2 \delta-1)}\|f\|_{\delta}\|k u\|_{-\delta},
\end{aligned}
$$

which, together with Corollary 4.3, gives (5.1). ॥

In the next theorem an improved estimate for $u=R(z) f$ will be given.

Theorem 5.2. Suppose that Assumption 2.1 holds with $N \geq 3$. Let $1 / 2<\delta \leq 1$. Let $u=R(z) f$, where $f \in L_{2, \delta}\left(\mathbf{R}^{N}\right)$ and $z=\lambda+i \eta$ with $\lambda \geq 0, \eta \neq 0$. Then there exists a positive constant $C=C(\delta)$ such that

$$
\int_{E_{s}}(1+r)^{-2 \delta}|u|^{2} d x \leq C(1+s)^{-2(2 \delta-1)} \frac{1}{|z|}\|f\|_{\delta}^{2} \quad(s \geq 0) .
$$

The constant $C=C(\delta)$ is independent of $f$ and $z$ satisfying the above conditions and $s \geq 0$.

Proof. Multiply both sides of the equation $\mu f=-\Delta u-k^{2} u$ by $a \alpha \bar{u}$, integrate over $B_{r}$ and take the imaginary part, where $\alpha(x)=1 / \sqrt{\mu(x)}$ again. 
Then we have

$$
\begin{aligned}
\int_{B_{r}} a \alpha \mu \operatorname{Im}(f \bar{u}) d x & =-\int_{S_{r}} a \alpha \operatorname{Im}\left(\frac{\partial u}{\partial r} \bar{u}\right) d S \\
& -\int_{B_{r}} 2 a^{2} b \alpha|u|^{2} d x \\
& \leq-\int_{S_{r}} a \alpha \operatorname{Im}\left(\frac{\partial u}{\partial r} \bar{u}\right) d S
\end{aligned}
$$

where we have noted that (5.2) holds with $\bar{k}$ replaced by $a$. Since

$$
\begin{aligned}
\left|\mathcal{D}_{r} u\right|^{2} & =\left|\frac{\partial u}{\partial r}+\frac{N-1}{2 r}+b u\right|^{2}+a^{2}|u|^{2}-2 a \operatorname{Im}\left(\frac{\partial u}{\partial r} \bar{u}\right) \\
& \geq a^{2}|u|^{2}-2 a \operatorname{Im}\left(\frac{\partial u}{\partial r} \bar{u}\right),
\end{aligned}
$$

we have from (5.8)

$$
\int_{S_{r}} a^{2} \alpha|u|^{2} d S \leq \int_{S_{r}} \alpha\left|\mathcal{D}_{r} u\right|^{2} d S-2 \int_{B_{r}} a \alpha \mu \operatorname{Im}(f \bar{u}) d x
$$

and hence, by the use of the first relation of (4.3), it follows that

$$
\int_{S_{r}}|u|^{2} d S \leq \frac{4}{e(z)^{2} \mu_{0}} \int_{S_{r}}\left|\mathcal{D}_{r} u\right|^{2} d S+\frac{4 M_{0}}{e(z) \sqrt{\mu_{0}}}\|f\|_{\delta}\|u\|_{-\delta}
$$

with $e(z)=\sqrt{2(|z|+\lambda)}$. Multiply both sides of $(5.11)$ by $(1+r)^{-2 \delta}$ and integrate on $(s, \infty)$. Then we see that, for $s \geq 0$,

$$
\begin{aligned}
\int_{E_{s}} & (1+r)^{-2 \delta}|u|^{2} d x \\
\leq & \frac{4}{e(z)^{2} \mu_{0}}(1+s)^{-2(2 \delta-1)} \int_{E_{s}}(1+r)^{2 \delta-2}\left|\mathcal{D}_{r} u\right|^{2} d x \\
& +\frac{4 M_{0}}{e(z)(2 \delta-1) \sqrt{\mu_{0}}}(1+s)^{-(2 \delta-1)}\|f\|_{\delta}\|u\|_{-\delta} .
\end{aligned}
$$

Note that $e(z)^{-1} \leq 1 / \sqrt{2|z|}$. Then, by setting $s=0$ in (5.12) and using Theorem 4.1, it follows that there exists a positive constant $C_{1}=C_{1}(\delta)$ such that

$$
\|u\|_{-\delta}^{2} \leq \frac{C_{1}}{|z|}\|f\|_{\delta}^{2}
$$

The estimate (5.7) is obtained from (5.12), (5.13) and Theorem 4.1. \| 
The following corollary is obtained easily when Theorems 5.1 is combined with Theorem 5.2.

Corollary 5.3. Suppose that Assumption 2.1 holds with $N \geq 3$. Let $1 / 2<\delta \leq 1$. Let $u=R(z) f$, where $f \in L_{2, \delta}\left(\mathbf{R}^{N}\right)$ and $z=\lambda+i \eta$ with $\lambda \geq 0, \eta \neq 0$. Then there exists a positive constant $C=C(\delta)$ such that

$$
\begin{aligned}
\int_{E_{s}} & (1+r)^{-2 \delta}\left(|\nabla u|^{2}+|k|^{2}|u|^{2}\right) d x \\
& \leq C\left(1+\frac{1}{\sqrt{|z|}}+\frac{1}{|z|}\right)(1+s)^{-(2 \delta-1)}\|f\|_{\delta}^{2} \quad(s \geq 1),
\end{aligned}
$$

where $E_{s}=\left\{x \in \mathbf{R}^{N}:|x|>s\right\}$, and \|\|$_{t}$ is the norm of $L_{2, t}\left(\mathbf{R}^{N}\right)$. The constant $C=C(\delta)$ is independent of $f$ and $z$ satisfying the above conditions and $s \geq 1$.

Now we are in a position to show some estimate of the operator norm of $R(z)$. For $0<c<d<\infty$ a subset $J_{ \pm}(c, d)$ of $\mathbf{C}$ are defined by

$$
\left\{\begin{array}{l}
J_{+}(c, d)=\{z=\lambda+i \eta: c \leq \lambda \leq d, 0<\eta \leq 1\}, \\
J_{-}(c, d)=\{z=\lambda+i \eta: c \leq \lambda \leq d,-1 \leq \eta<0\} .
\end{array}\right.
$$

Let $t \in \mathbf{R}$. The weighted Sobolev spaces $H_{t}^{j}\left(\mathbf{R}^{N}\right), j=1,2$, are defined as the completion of $C_{0}^{\infty}\left(\mathbf{R}^{N}\right)$ by the norms

$$
\|u\|_{1, t}=\left[\int_{\mathbf{R}^{N}}(1+r)^{2 t}\left(|\nabla u|^{2}+|u(x)|^{2}\right) d x\right]^{1 / 2},
$$

and

$$
\|u\|_{2, t}=\left[\int_{\mathbf{R}^{N}}(1+r)^{2 t} \sum_{|\gamma| \leq 2}\left|\partial^{\gamma} u\right|^{2} d x\right]^{1 / 2},
$$

respectively, where

$$
\left\{\begin{array}{l}
\gamma=\left(\gamma_{1}, \gamma_{2}, \cdots, \gamma_{N}\right) \\
|\gamma|=\gamma_{1}+\gamma_{2}+\cdots+\gamma_{N} \\
\partial^{\gamma} u=\left(\partial_{1}\right)^{\gamma_{1}} \cdots\left(\partial_{N}\right)^{\gamma_{N}} u \quad\left(\partial_{j}=\partial / \partial x_{j}\right) .
\end{array}\right.
$$

The inner product and norm of $H_{t}^{j}\left(\mathbf{R}^{N}\right)$ will be denoted by $(,)_{j, t}$ and \|\|$_{j, t}$. For an operator $T$, the operator norm in $\mathbf{B}\left(H_{s}^{j}\left(\mathbf{R}^{N}\right), H_{t}^{\ell}\left(\mathbf{R}^{N}\right)\right)$ will be denoted by $\|T\|_{(j, s)}^{(\ell, t)}$, where $j, \ell=0,1,2, s, t \in \mathbf{R}$, and we set

$$
H_{s}^{0}\left(\mathbf{R}^{N}\right)=L_{2, s}\left(\mathbf{R}^{N}\right)
$$


Theorem 5.4. Suppose that Assumption 2.1 holds with $N \geq 3$. Let $1 / 2<\delta \leq 1$. Let $R(z)$ be the resolvent of $H$.

(i) Then there exists a positive constant $C=C(\delta)$ such that

$$
\|R(z)\|_{(0, \delta)}^{(0,-\delta)} \leq \frac{C}{\sqrt{|z|}}
$$

for $z=\lambda+i \eta \in \mathbf{C}$ with $\lambda \geq 0$ and $\eta \neq 0$.

(ii) Let $0<c<d<\infty$ and let $J_{ \pm}(c, d)$ be as above. Then there exists a positive constant $C=C(\delta, c, d)$ such that

$$
\|R(z)\|_{(0, \delta)}^{(2,-\delta)} \leq C
$$

for $z \in J_{+}(c, d) \cup J_{-}(c, d)$.

Proof. (i) directly follows from (5.10) in Theorem 5.3 with $s=0$. It follows from (4.2) in Theorem 4.1 that

$$
\begin{aligned}
\int_{E_{1}} & (1+r)^{-2 \delta}|\nabla u|^{2} d x \\
& \leq 2 \int_{E_{1}}(1+r)^{-2 \delta}|\mathcal{D} u|^{2} d x \\
& \quad+2 \int_{E_{1}}(1+r)^{-2 \delta}\left|\frac{N-1}{2 r} \widetilde{x} u-i k \widetilde{x} u\right|^{2} d x \\
& \leq 2 \int_{E_{1}}(1+r)^{2 \delta-2}|\mathcal{D} u|^{2} d x \\
& \quad+4 \int_{E_{1}}\left(\left(\frac{N-1}{2}\right)^{2}+|k|^{2}\right)(1+r)^{-2 \delta}|u|^{2} d x \\
\leq & C_{2}(\delta)\|f\|_{\delta}^{2}+C_{3}(\delta, c, d)\|u\|_{-\delta}^{2}
\end{aligned}
$$

with positive constants $C_{2}=C_{2}(\delta)$ and $C_{3}=C_{3}(\delta, c, d)$, where $u=R(z) f$ with $f \in L_{2, \delta}\left(\mathbf{R}^{N}\right)$ and $z \in J_{+}(c, d) \cup J_{-}(c, d)$. Since the $L_{2}\left(B_{1}\right)$-norm of $\nabla u$ can be evaluated by the interior estimate, we obtain from (5.22) and (5.20)

$$
\|u\|_{1,-\delta} \leq C_{4}(\delta, c, d)\|f\|_{2, \delta}
$$

with a positive constant $C_{4}=C_{4}(\delta, c, d)$, which implies that

$$
\|R(z)\|_{(0, \delta)}^{(1,-\delta)} \leq C_{5}(\delta, c, d) \quad\left(z \in J_{+}(c, d) \cup J_{-}(c, d)\right)
$$

with a constant $C_{5}=C_{5}(\delta, c, d)$. Using the relation $\Delta u=-\mu f-k^{2} u$, we have from (5.20)

$$
\|\Delta u\|_{-\delta} \leq C_{6}(\delta, c, d)\|f\|_{\delta}
$$


with a constant $C_{6}=C_{6}(\delta, c, d)$. The inequality (5.21) follows from (5.23) and (5.24) ([8], Proposition A.2), which completes the proof. \|

\section{§6. Limiting absorption principle.}

By the use of the results established in $\S 3, \S 4$ and $\S 5$, we can show the limiting absorption principle for the operator $H$ in $\mathbf{R}^{N}$ with $N \geq 3$ using the arguments used to prove the limiting absorption principle for the Schrödinger operator (e.g., [11], [7]).

First we shall define the boundary value $R^{ \pm}(\lambda), \lambda>0$, of the resolvent $R(z)$ when $z=\lambda+i \eta \rightarrow \lambda$.

Theorem 6.1. Suppose that Assumption 2.1 holds with $N \geq 3$. Let $1 / 2<\delta \leq 1$. Let $f \in L_{2, \delta}\left(\mathbf{R}^{N}\right)$ and let $\lambda>0$. Then there exist

$$
\left\{\begin{array}{l}
\lim _{\eta \downarrow 0} R(\lambda+i \eta) f=u_{+}(\cdot, \lambda, f), \\
\lim _{\eta \downarrow 0} R(\lambda-i \eta) f=u_{-}(\cdot, \lambda, f),
\end{array}\right.
$$

in $H_{-\delta}^{2}\left(\mathbf{R}^{N}\right)$, where $H_{-\delta}^{2}\left(\mathbf{R}^{N}\right)$ is given in $\S 5$, and $u_{+}(\cdot, \lambda, f) \quad\left[\right.$ or $\left.u_{-}(\cdot, \lambda, f)\right]$ is a unique solution of the equation

$$
\left\{\begin{array}{l}
-\mu(x)^{-1} \Delta u-\lambda u=f \\
\left\|\mathcal{D}^{(+)} u\right\|_{\delta-1}<\infty \quad\left[\text { or }\left\|\mathcal{D}^{(-)} u\right\|_{\delta-1}<\infty\right]
\end{array}\right.
$$

with

$$
\mathcal{D}^{( \pm)} u=\nabla u+\{(N-1) /(2 r)\} \widetilde{x} u \mp i \sqrt{\lambda \mu(x)} \widetilde{x} u .
$$

Proof. For each $n=1,2, \cdots$ let $z_{n}=\lambda+i \eta_{n}$, where $\eta_{n}>0$ and $\eta_{n} \downarrow 0$ as $n \rightarrow \infty$. Set $u_{n}=R\left(z_{n}\right) f, n=1,2, \cdots$. Then, in view of Corollary 5.3 and Theorems 5.4, we see that not only the sequence $\left\{u_{n}\right\}$ is a bounded set in $H_{-\delta}^{2}\left(\mathbf{R}^{N}\right)$ but also $\left\|u_{n}\right\|_{1,-\delta, E_{s}}$ is uniformly small for $n$ as $s \rightarrow \infty$, where

$$
\left\|u_{n}\right\|_{1,-\delta, E_{s}}^{2}=\int_{E_{s}}(1+|x|)^{-2 \delta}\left|u_{n}\right|^{2} d x .
$$

Therefore, by the Rellich selection theorem, $\left\{u_{n}\right\}$ has a subsequence which converges to a limit function $u_{0}$ in $H_{-\delta}^{2}\left(\mathbf{R}^{N}\right)$ (see, e.g., [8], Proposition A.3). Since $u_{0}$ turns out to be a unique solution of the equation $-\Delta u-k^{2} u=$ $\mu f$ with radiation condition $\left\|\mathcal{D}^{(+)} u\right\|_{\delta-1}<\infty$, i.e., $u_{0}=u_{+}(\cdot, \lambda, f)$, it follows that the sequence $\left\{u_{n}\right\}$ itself converges to $u_{0}=u_{+}(\cdot, \lambda, f)$ in $H_{-\delta}^{2}\left(\mathbf{R}^{N}\right)$. 
The existence of the first limit of (6.1) follows from the above argument. The existence of the second limit of (6.1) can be proved in the same way. \|

Definition 6.2. Let $\lambda>0$. Then the operators $R_{ \pm}(\lambda)$ are defined by

$$
\left\{\begin{array}{l}
R_{+}(\lambda): L_{2, \delta}\left(\mathbf{R}^{N}\right) \ni f \longmapsto u_{+}(\cdot, \lambda, f) \in H_{-\delta}^{2}\left(\mathbf{R}^{N}\right), \\
R_{-}(\lambda): L_{2, \delta}\left(\mathbf{R}^{N}\right) \ni f \longmapsto u_{-}(\cdot, \lambda, f) \in H_{-\delta}^{2}\left(\mathbf{R}^{N}\right),
\end{array}\right.
$$

Let $D_{ \pm} \subset \mathbf{C}$ be given by

$$
\left\{\begin{array}{l}
D_{+}=\{z=\lambda+i \eta: \lambda>0, \eta \geq 0\} \\
D_{-}=\{z=\lambda+i \eta: \lambda>0, \eta \leq 0\}
\end{array}\right.
$$

Then the resolvent $R(z)$ will be extended on each of $D_{ \pm}$by the use of $R_{ \pm}(\lambda)$, i.e., for $z \in D_{+}$we set

$$
R(\lambda+i \eta)= \begin{cases}R(\lambda+i \eta) & (\lambda>0, \eta>0), \\ R_{+}(\lambda) & (\lambda>0, \eta=0),\end{cases}
$$

and for $z \in D_{-}$we set

$$
R(\lambda+i \eta)= \begin{cases}R(\lambda+i \eta) & (\lambda>0, \eta<0) \\ R_{-}(\lambda) & (\lambda>0, \eta=0)\end{cases}
$$

For $0<c<d<\infty$ let $J_{ \pm}(c, d)$ be as in (5.15). The closure $\bar{J}_{ \pm}(c, d)$ are given by

$$
\left\{\begin{array}{l}
\bar{J}_{+}(c, d)=\{z=\lambda+i \eta: c \leq \lambda \leq d, 0 \leq \eta \leq 1\} \subset D_{+}, \\
\bar{J}_{-}(c, d)=\{z=\lambda+i \eta: c \leq \lambda \leq d,-1 \leq \eta \leq 0\} \subset D_{-} .
\end{array}\right.
$$

For $\lambda \in D_{+} \cap(0, \infty)$ [or $\left.D_{-} \cap(0, \infty)\right]$, $\mathcal{D} u$ should be interpreted as $\mathcal{D}^{(+)}[$or $\left.\mathcal{D}^{(-)}\right]$.

From Theorems $6.1,5.1,5.2,5.4$ and Corollary 5.3, we easily see the following:

Theorem 6.3. Let $1 / 2<\delta \leq 1$. Suppose Assumption 2.1 holds with $N \geq 3$. Let $R(z)$ be extended on each of $D_{+}$and $D_{-}$. 
(i) Then there exists a positive constant $C=C(\delta)$ such that

$$
\left\{\begin{array}{l}
\int_{E_{s}}(1+r)^{-2 \delta}|R(z) f|^{2} d x \leq \frac{C^{2}}{|z|}(1+s)^{-2(2 \delta-1)}\|f\|_{\delta}^{2} \\
\|R(z)\|_{(0, \delta)}^{(0,-\delta)} \leq \frac{C}{\sqrt{|z|}} \quad\left(s \geq 0, f \in L_{2, \delta}\left(\mathbf{R}^{N}\right)\right), \\
\|\mathcal{D} R(z) f\|_{\delta-1} \leq C\|f\|_{\delta} \quad\left(z \in D_{+} \cup D_{-}\right),
\end{array}\right.
$$

(ii) For $0<c<d<\infty$ there exists a positive constant $C=C(c, d, \delta)$ such that, for $z \in \bar{J}_{+}(c, d) \cup \bar{J}_{-}(c, d)$,

$$
\left\{\begin{array}{r}
\|R(z)\|_{(0, \delta)}^{(2,-\delta)} \leq C, \\
\int_{E_{s}}(1+r)^{-2 \delta}\left(|\nabla R(z) f|^{2}+|k|^{2}|R(z) f|^{2}\right) d x \\
\leq C^{2}(1+s)^{-(2 \delta-1)}\|f\|_{\delta}^{2} \\
\quad\left(s \geq 1, f \in L_{2, \delta}\left(\mathbf{R}^{N}\right)\right) .
\end{array}\right.
$$

The next proposition will be used when we prove continuity of $R(z)$ with respect to $z$ and the compactness of the operator $R(z)$.

Proposition 6.4. Let $1 / 2<\delta \leq 1$. Suppose that Assumption 2.1 holds with $N \geq 3$. Let $R(z)$ be extended on each of $D_{+}$and $D_{-}$. Let $\left\{f_{n}\right\}$ be a sequence in $L_{2, \delta}\left(\mathbf{R}^{N}\right)$ such that

$$
f_{n} \rightarrow f_{0} \quad \text { weakly in } L_{2, \delta}\left(\mathbf{R}^{N}\right)
$$

as $n \rightarrow \infty$, and let $\left\{z_{n}\right\} \subset \bar{J}_{+}(c, d)\left[\right.$ or $\left.\left\{z_{n}\right\} \subset \bar{J}_{+}(c, d)\right]$ with $0<c<d<$ $\infty$ such that

$$
z_{n} \rightarrow z_{0} \quad(n \rightarrow \infty) .
$$

Then there exists a sequence $\left\{n_{k}\right\}_{k=1}^{\infty}$ of positive integers such that

$$
n_{1}<n_{2}<n_{3}<\cdots<n_{k}<\cdots \rightarrow \infty
$$

and

$$
u_{n_{k}} \rightarrow R\left(z_{0}\right) f_{0} \quad \text { in } H_{-\delta}^{1}\left(\mathbf{R}^{N}\right)
$$

as $k \rightarrow \infty$, where $u_{n_{k}}=R\left(z_{n_{k}}\right) f_{n_{k}}$. 
Proof. We are going to give the proof for the case that $z_{0}=\lambda_{0} \in$ $[c, d]$ and $\left\{z_{n}\right\} \subset \bar{J}_{+}(c, d)$. The case that $z_{0}$ is not a real number can be treated more easily. Set $u_{n}=R\left(z_{n}\right) f_{n}$. Using Theorem 6.3 and proceeding as in the proof of Theorem 6.1, we see that there exists a subsequence of $\left\{u_{n}\right\}$ which converges to a limit function $u_{0}$ in $H_{-\delta}^{1}\left(\mathbf{R}^{N}\right)$. Then it is easy to show that $u_{0} \in H_{-\delta}^{2}\left(\mathbf{R}^{N}\right)$ and that $u_{0}=u_{+}\left(\cdot, \lambda_{0}, f_{0}\right)=R\left(\lambda_{0}\right) f_{0}$, which completes the proof.

The following properties of the extended resolvent $R(z)$ follows directly from the above proposition.

Theorem 6.5. Let $1 / 2<\delta \leq 1$. Suppose that Assumption 2.1 holds with $N \geq 3$. Let $R(z)$ be extended on each of $D_{+}$and $D_{-}$.

(i) Then the extended resolvent $R(z)$ is a $\mathbf{B}\left(L_{2, \delta}\left(\mathbf{R}^{N}\right), H_{-\delta}^{2}\left(\mathbf{R}^{N}\right)\right)$-valued continuous function on each of $D_{+}$and $D_{-}$.

(ii) For any $z \in D_{+}\left[\right.$or $\left.D_{-}\right], R(z)$ is a compact operator from $L_{2, \delta}\left(\mathbf{R}^{N}\right)$ into $H_{-\delta}^{1}\left(\mathbf{R}^{N}\right)$.

Proof. (I) The proof of (i). Suppose that there is $z_{0} \in D_{+}$at which $R(z)$ is not continuous in the topology of $\mathbf{B}\left(L_{2, \delta}\left(\mathbf{R}^{N}\right), H_{-\delta}^{1}\left(\mathbf{R}^{N}\right)\right)$. We may assume that $z_{0}=\lambda_{0}>0$, since the other case can be handled more easily. Then there exist $\epsilon_{0}>0$ and sequences $\left\{z_{n}\right\} \subset D_{+},\left\{f_{n}\right\} \subset L_{2, \delta}\left(\mathbf{R}^{N}\right)$ and $f_{0} \in L_{2, \delta}\left(\mathbf{R}^{N}\right)$ such that

$$
\left\{\begin{array}{lc}
z_{n} \rightarrow \lambda_{0} & (n \rightarrow \infty), \\
\left\|f_{n}\right\|_{\delta}=1 & (n=1,2, \cdots), \\
f_{n} \rightarrow f_{0} & \text { weakly in } L_{2, \delta}\left(\mathbf{R}^{N}\right), \\
\left\|R\left(\lambda_{0}\right) f_{n}-R\left(z_{n}\right) f_{n}\right\|_{1,-\delta} \geq \epsilon_{0}
\end{array}\right.
$$

Applying Proposition 6.4 for the sequence $\left\{R\left(\lambda_{0}\right) f_{n}\right\}$, we see that there exists a subsequence $\left\{f_{n_{k}}\right\}$ of $\left\{f_{n}\right\}$ such that

$$
R\left(\lambda_{0}\right) f_{n_{k}} \rightarrow R\left(\lambda_{0}\right) f_{0} \quad \text { in } H_{-\delta}^{1}\left(\mathbf{R}^{N}\right)
$$

as $k \rightarrow \infty$. Apply Proposition 6.4 again for the sequence $\left\{R\left(z_{n_{k}}\right) f_{n_{k}}\right\}_{k=1}^{\infty}$ to see that there is a subsequence $\left\{R\left(z_{n_{k_{p}}}\right) f_{n_{k_{p}}}\right\}_{p=1}^{\infty}$ such that

$$
R\left(z_{n_{k_{p}}}\right) f_{n_{k_{p}}} \rightarrow R\left(\lambda_{0}\right) f_{0} \quad \text { in } H_{-\delta}^{1}\left(\mathbf{R}^{N}\right) .
$$


as $p \rightarrow \infty$. Therefore it follows from (6.17) and (6.18) that

$$
\begin{aligned}
& \left\|R\left(\lambda_{0}\right) f_{n_{k_{p}}}-R\left(z_{n_{k_{p}}}\right) f_{n_{k_{p}}}\right\|_{1,-\delta} \\
& \quad \leq\left\|R\left(\lambda_{0}\right) f_{n_{k_{p}}}-R\left(\lambda_{0}\right) f_{0}\right\|_{1,-\delta} \\
& \quad \quad+\left\|R\left(\lambda_{0}\right) f_{0}-R\left(z_{n_{k_{p}}}\right) f_{n_{k_{p}}}\right\|_{1,-\delta} \\
& \quad \rightarrow 0 \quad
\end{aligned}
$$

as $p \rightarrow \infty$, which contradicts the fourth relation of (6.16). Thus we have shown that $R(z)$ is a $\mathbf{B}\left(L_{2, \delta}\left(\mathbf{R}^{N}\right), H_{-\delta}^{1}\left(\mathbf{R}^{N}\right)\right)$-valued continuous function on each of $D_{+}$and $D_{-}$.

(II) Proof of (i) (continued). Let $z, z_{0} \in D_{+}\left[\right.$or $\left.z, z_{0} \in D_{-}\right]$. The continuity of $R(z)$ in $\mathbf{B}\left(L_{2, \delta}\left(\mathbf{R}^{N}\right), H_{-\delta}^{1}\left(\mathbf{R}^{N}\right)\right)$ is combined with the relation

$$
\begin{aligned}
\Delta R(z)-\Delta & R\left(z_{0}\right) \\
& =-z \mu R(z)+z_{0} \mu R\left(z_{0}\right) \\
& =\left(z_{0}-z\right) R\left(z_{0}\right)+z\left(R\left(z_{0}\right)-R(z)\right) \rightarrow 0
\end{aligned}
$$

in $\mathbf{B}\left(L_{2, \delta}\left(\mathbf{R}^{N}\right), L_{2,-\delta}\left(\mathbf{R}^{N}\right)\right)$ as $z \rightarrow z_{0}$ to obtain the continuity of $R(z)$ in $\mathbf{B}\left(L_{2, \delta}\left(\mathbf{R}^{N}\right), H_{-\delta}^{1}\left(\mathbf{R}^{N}\right)\right.$ ) (cf., e.g., [8], Proposition A.3 in Appendix A.2). This completes the proof of (i).

(III) Proof of (ii). Let $\left\{f_{n}\right\}$ be a bounded sequence in $L_{2, \delta}\left(\mathbf{R}^{N}\right)$. We may assume with no loss of generality that the sequence $\left\{f_{n}\right\}$ converges weakly in $L_{2, \delta}\left(\mathbf{R}^{N}\right)$. The weak limit will be denoted by $f_{0}$. Then, applying Proposition 6.4 , we see that there exists a subsequence $\left\{f_{n_{k}}\right\}_{k=1}^{\infty}$ such that

$$
R(z) f_{n_{k}} \rightarrow R(z) f_{0} \quad \text { in } H_{-\delta}^{1}\left(\mathbf{R}^{N}\right)
$$

as $k \rightarrow \infty$, which completes the proof of (ii). $\|$

(i) of Theorem 6.5 and the spectral formula for self-adjoint operators are combined to give

Corollary 6.6 Suppose that Assumption 2.1 holds with $N \geq 3$. Then the selfadjoint operator $H$ is absolutely continuous on the interval $(0, \infty)$.

\section{$\S 7$. The operator $H$ in $\mathbf{R}^{2}$.}

In the two dimensional case, the constant $c_{N}$ given by (3.8) takes the value $c_{2}=-1 / 4<0$ although $c_{N} \geq 0$ for all $N \geq 3$. Because of this, we 
are going to make some technical changes in the theory which was dveloped in $\S 3 \sim \S 6$. Also we should note that $u /|x|$ is not necessarily integrable around $x=0$ for $u \in H^{2}\left(\mathbf{R}^{2}\right)_{\text {loc }}$ although we have

$$
\frac{u}{|x|^{1 / 2}} \in L_{2}\left(\mathbf{R}^{2}\right)_{\text {loc }} \quad\left(u \in H^{2}\left(\mathbf{R}^{2}\right)_{\text {loc }}\right)
$$

since $u \in H^{2}\left(\mathbf{R}^{2}\right)_{\text {loc }}$ is a continuous function on $\mathbf{R}^{2}$. We are going to use Notation 3.1 (with $N=2$ ) throughout this section.

7.1. Uniqueness of the solution.

The uniqueness theorem takes the following form:

Theorem 7.1. Suppose that Assumption 2.1 with $N=2$ holds. Let $u \in H^{2}\left(\mathbf{R}^{N}\right)_{\text {loc }}$ be a solution of the homogeneous equation

$$
-\mu(x)^{-1} \Delta u-\lambda u=0 \quad(\lambda>0)
$$

on $\mathbf{R}^{2}$ such that

$$
\liminf _{R \rightarrow \infty} R^{\alpha} \int_{S_{R}}\left(\left|\frac{\partial u}{\partial r}\right|^{2}+|u|^{2}\right) d S=0
$$

with $\alpha>0$. Then $u$ is identically zero.

Proof. (I) Note that Proposition 3.3 and Lemma 3.4 are true for the case of $N=2$ without any change. Also Lemma 3.5 is true if we add a condition that

$$
\frac{\partial \varphi}{\partial r}=O(r)
$$

as $r \downarrow 0$.

(II) We may assume with no loss of generality that $0<\alpha \leq 1$. Let

$$
\varphi(x)= \begin{cases}|x|^{2} & \left(0 \leq|x| \leq r_{0}\right), \\ r_{0}^{2-\alpha}|x|^{\alpha} & \left(|x|>r_{0}\right)\end{cases}
$$

in (3.7), where $r_{0}>0$ will be determined later. Proceeding as in the proof of Theorem 3.2, we have from (3.7), for any $R>r_{0}>r>0$, 


$$
\begin{aligned}
\int_{B_{R}} \frac{1}{2} & \frac{\partial \varphi}{\partial r} k^{2}|u|^{2} d x+\int_{B_{r R}}\left(\frac{\varphi}{r}-2^{-1} \frac{\partial \varphi}{\partial r}\left(|\nabla u|^{2}-\left|\frac{\partial u}{\partial r}\right|^{2}\right) d x\right. \\
& \quad-\frac{1}{4} \int_{B_{r R}} r^{-2}\left(\frac{\varphi}{r}-2^{-1} \frac{\partial \varphi}{\partial r}\right)|u|^{2} d x \\
\leq & 2^{-1} \sum_{\ell=1}^{2} \int_{\partial \Omega_{\ell} \cap B_{r R}} \varphi|k|^{2}(\widetilde{x} \cdot n)|u|^{2} d S \\
& +2^{-1} r_{0}^{2-\alpha} R^{\alpha} \int_{S_{R}}\left(2\left|\mathcal{D}_{r} u\right|^{2}-|\mathcal{D} u|^{2}+\frac{1}{4} r^{-2}|u|^{2} \operatorname{Im}\left(k \frac{\partial u}{\partial n} \bar{u}\right)\right) d S \\
& -2^{-1} r^{2} \int_{S_{r}}\left(2\left|\mathcal{D}_{r} u\right|^{2}-|\mathcal{D} u|^{2}+\frac{1}{4} r^{-2}|u|^{2}\right) d S \\
& +\int_{B_{r}} \frac{1}{2} r^{2}|\mathcal{D} u|^{2} d x+\sum_{\ell=1}^{2} \int_{\partial \Omega_{\ell} \cap B_{r}} r^{2} \operatorname{Im}\left(k \frac{\partial u}{\partial r} \bar{u}\right) d S .
\end{aligned}
$$

The left-hand side and right-hand side of (7.6) will be denoted by $K_{L}$ and $K_{R}$, respectively.

(III) As in the proof of Theorem 3.2, we see that the second term of $K_{L}$ is nonnegative: Thus we have

$$
\begin{aligned}
& K_{L} \geq \int_{B_{r_{0}}} r k^{2}|u|^{2} d x+\int_{B_{r_{0} R}}\left(\frac{1}{2} \frac{\partial \varphi}{\partial r} k^{2}-\frac{1}{4} r^{-2}\left(\frac{\varphi}{r}-2^{-1} \frac{\partial \varphi}{\partial r}\right)\right)|u|^{2} d x \\
&=\int_{B_{r_{0}}} r k^{2}|u|^{2} d x \\
& \quad+r_{0}^{2-\alpha} \int_{B_{r_{0} R}} r^{\alpha-1}\left(\frac{\alpha}{2} k^{2}-\frac{1}{4}\left(1-\frac{\alpha}{2}\right) r^{-2}\right)|u|^{2} d x .
\end{aligned}
$$

Choose $r_{0}=r_{0}(k, \alpha)$ so large that

$$
\frac{\alpha}{2} k^{2}-\frac{1}{4}\left(1-\frac{\alpha}{2}\right) r^{-2} \geq \frac{\alpha k^{2}}{4} \quad\left(r \geq r_{0}\right)
$$

Then we have

$$
K_{L} \geq \int_{B_{r_{0}}} r k^{2}|u|^{2} d x+r_{0}^{2-\alpha} \int_{B_{r_{0} R}} r^{\alpha-1} \frac{\alpha k^{2}}{4}|u|^{2} d x .
$$

(IV) We cn proceed as in the proof Theorem 3.2 to see that the first term of $K_{R}$ is nonpositive, the fourth and fifth term go to 0 as $r \downarrow 0$, and the 
third term goes to 0 as $r \downarrow 0$ along an appropriate sequence. Therefore, after evaluating the third term using $|u|$ and $|\partial u / \partial u|$, we obtain

$$
\begin{gathered}
\int_{B_{r_{0}}} r k^{2}|u|^{2} d x+r_{0}^{2-\alpha} \int_{B_{r_{0} R}} r^{\alpha-1} \frac{\alpha k^{2}}{4}|u|^{2} d x \\
\leq C R^{\alpha} \int_{S_{R}}\left(\left|\frac{\partial u}{\partial r}\right|^{2}+|u|^{2}\right) d S
\end{gathered}
$$

with $C=C(\lambda, \alpha)$, and hence it follows from the condition (7.3) that

$$
\int_{B_{r_{0}}} r k^{2}|u|^{2} d x+r_{0}^{2-\alpha} \int_{E_{r_{0}}} r^{\alpha-1} \frac{\alpha k^{2}}{4}|u|^{2} d x=0,
$$

where $E_{r_{0}}=\left\{x \in \mathbf{R}^{2}:|x|>r_{0}\right\}$, i.e., $u$ is identically zero, which completes the proof. $\|$

Since we have established Theorem 7.1, a two dimensional version of Theorem 3.2, we can easily see that each of Corollary 3.6, Theorem 3.7 and Corollary 3.8 has its two dimensional version only by replacing the conditions (3.24), (3.25), and (3.35) by

$$
\begin{gathered}
\liminf _{R \rightarrow \infty} R^{\alpha} \int_{S_{R}}\left|\mathcal{D}_{r}^{( \pm)} u\right|^{2} d S=0, \\
\liminf _{R \rightarrow \infty} R^{\alpha} \int_{S_{R}}\left|\frac{\partial u}{\partial r} \mp i k u\right|^{2} d S=0,
\end{gathered}
$$

and

$$
\left\{\begin{array}{l}
\int_{E_{R}} r^{-1+\alpha}\left|\mathcal{D}_{r}^{( \pm)} u\right|^{2} d x<\infty, \\
\int_{E_{R}} r^{-1+\alpha}\left|\frac{\partial u}{\partial r} \mp i k u\right|^{2}, d x<\infty,
\end{array}\right.
$$

with $\alpha>0$. We do not take the trouble to write down these two dimensional versions since they are now quite obvious.

7.2. The evaluation of $\mathcal{D} u$.

Consider $u$ given by

$$
\left\{\begin{array}{l}
u=R(z) f, \\
z=\lambda+i \eta \\
f \in L_{2, \delta}\left(\mathbf{R}^{2}\right) .
\end{array} \quad(\lambda \geq 0, \eta \neq 0),\right.
$$


Theorem 7.2. Assume Assumption 2.1 with $N=2$. Let $1 / 2<\delta \leq 1$. Let $0<c<d<\infty$ and let $J_{ \pm}(c, d)$ be as in (5.15). Let $u$ be given by (7.15) with $z \in J_{+}(c, d) \cup J_{-}(c, d)$. Then there exists a positive constant $C=$ $C(c, d, \mu, \delta)$ such that

$$
\|\mathcal{D} u\|_{\delta-1 . *} \leq C\left(\|f\|_{\delta}+\|u\|_{-\delta}\right)
$$

where

$$
\|v\|_{t, *}^{2}=\int_{B_{1}}|x||v(x)|^{2} d x+\int_{E_{1}}(1+|x|)^{2 t}|v(x)|^{2} d x .
$$

Proof. Set $\varphi(x)=\xi(|x|) / \sqrt{\mu(x)}$ in (3.7), where

$$
\xi(r)= \begin{cases}\frac{1}{2} r^{2} & (r \leq 1 / 2), \\ \frac{1}{2^{2 \delta}}(1+r)^{2 \delta-1} & (r \geq 1) .\end{cases}
$$

We can evaluate each term in (3.7) in quite a similar manner as in the Proof of theorem 4.1 except the fourth term $I_{L 4}$ of the left-hand side which is nonpositive in our case because $c_{2}=-1 / 4<0$. The term $-I_{L 4}$ can be evaluated as

$$
\begin{aligned}
-I_{L 4} & =\frac{1}{4} \int_{B_{1 / 2, R}} r^{-2}\left(\frac{\varphi}{r}-2^{-1} \frac{\partial \varphi}{\partial r}+b \varphi\right)|u|^{2} d x \\
& \leq C_{1}\|u\|_{-\delta}^{2}+C_{2} \int_{\mathbf{R}^{2}}|\eta \| u|^{2} d x,
\end{aligned}
$$

and the second term of the right-hand side of (7.19) is eavaluated as

$$
\int_{\mathbf{R}^{2}}|\eta||u|^{2} d x \leq C_{3}(|f|,|u|)_{0}
$$

(see, e.g., Eidus [6], [13], Lemma 2.1), where $C_{1}=C_{1}\left(\mu_{0}, \delta\right), C_{2}=C_{2}(c, d)$, and $C_{3}=C_{3}(c, d, \mu)$. Thus, using (7.19) and (7.21), we can proceed as in the proof of Theorem 4.1 to obtain (7.16), which completes the proof. \|

The following corollary is now obvious.

Corollary 7.3. Let $u=R(z) f$ be ae in Theorem 7.2. Then there exists a positive constant $C=C(c, d, \mu, \delta)$ such that

$$
\int_{E_{1}}(1+|x|)^{2 \delta-2}|\nabla u-i k \widetilde{x} u|^{2} d x \leq C\left(\|f\|_{\delta}^{2}+\|u\|_{-\delta}^{2}\right) .
$$


7.3. Boundedness of $R(z)$ and the limiting absorption principle.

The following theorem can be proved in quite the same manner as in the proof of Theorem 5.1.

Theorem 7.4. Suppose that Assumption 2.1 holds with $N=2$. Let $1 / 2<\delta \leq 1$. Let $0<c<d<\infty$ and let $J_{ \pm}(c, d)$ be as in (5.15). Let $u$ be given by (7.15) with $z \in J_{+}(c, d) \cup J_{-}(c, d)$. Then there exists a positive constant $C=C(c, d, \mu, \delta)$ such that

$$
\begin{aligned}
\int_{E_{s}}(1+r)^{-2 \delta} & \left(|\nabla u|^{2}+|k|^{2}|u|^{2}\right) d x \\
& \leq C(1+s)^{-(2 \delta-1)}\left(\|f\|_{\delta}^{2}+\|u\|_{-\delta}^{2}\right) \quad(s \geq 1)
\end{aligned}
$$

where $E_{s}=\left\{x \in \mathbf{R}^{2}:|x|>s\right\}$, and \|\|$_{t}$ is the norm of $L_{2, t}\left(\mathbf{R}^{2}\right)$.

Let $H_{t}^{j}\left(\mathbf{R}^{2}\right), j=1,2$, be defined in $\S 5$ with $N=2$. In order to obtain the counterpart of Theorem 5.4, we prepare

Proposition 7.5. Assume Assumption 2.1 with $N=2$. Let $1 / 2<\delta \leq$ 1. Let $0<c<d<\infty$ and let $J_{ \pm}(c, d)$ be as in (5.15). Let $u$ be given by (7.15) with $z \in J_{+}(c, d) \cup J_{-}(c, d)$. Then there exists a positive constant $C=C(c, d, \mu, \delta)$ such that

$$
\|u\|_{1,-\delta} \leq C\|f\|_{\delta}
$$

Proof. Suppose that (7.23) is not true. Then, for each $n=1,2, \cdots$, there exist $f_{n} \in L_{2, \delta}\left(\mathbf{R}^{2}\right)$ and $z_{n} \in J_{+}(c, d) \cup J_{-}(c, d)$ such that

$$
\left\{\begin{array}{l}
\left\|f_{n}\right\|_{\delta}<1 / n, \\
\left\|u_{n}\right\|_{1,-\delta}=1
\end{array}\right.
$$

where $u_{n}=R\left(z_{n}\right) f_{n}$. Here we may assume with no loss of generality that $z_{n}$ converges to $z_{0}$ which is in the closure of $J_{+}(c, d) \cup J_{-}(c, d)$. We consider the case that $z_{0}=\lambda \in[c, d]$ since the other case is much easier. Then, using the Rellich selection theorem and the equation

$$
-\Delta u_{n}-\mu(x) z_{n} u_{n}=\mu(x) f_{n}
$$

we see there exists a subsequence of $\left\{u_{n}\right\}$ which is a Cauchy sequence in $H^{1}\left(\mathbf{R}^{2}\right)_{\text {loc }}$. For the sake of simplicity of notation we denote the subsequence by 
$\left\{u_{n}\right\}$ again. In view of Theorem 7.4, $\left\{u_{n}\right\}$ is a Cauchy sequence in $H_{-\delta}^{1}\left(\mathbf{R}^{2}\right)$. Let $u_{0}$ be the limit function. We have $\left\|u_{0}\right\|_{1,-\delta}=1$. It is easy to see that $u_{0}$ is a (weak) solution of the homogeneous equation $-\Delta u_{0}-\mu(x) \lambda u_{0}=0$, and hence we have $u_{0} \in H_{-\delta}^{2}\left(\mathbf{R}^{2}\right)$. On the other hand, it follows from Theorem 7.2 that, for $n=1,2, \cdots$,

$$
\begin{aligned}
\left\|\mathcal{D} u_{n}\right\|_{\delta-1, E_{1}} & \leq C\left(\left\|f_{n}\right\|_{\delta}+\left\|u_{n}\right\|_{-\delta}\right) \\
& \leq C(1 / n+1) \leq 2
\end{aligned}
$$

and hence, by letting $n \rightarrow \infty$, we have $\left\|\mathcal{D} u_{0}\right\|_{\delta-1, E_{1}}<\infty$, i.e., $u_{0}$ satisfies the radiation condition. Therefore, by the two dimensional counterpart of Corollary 3.8 , we have $u_{0}=0$, which contradicts the fact that $\left\|u_{0}\right\|_{1,-\delta}=1$. This completes the proof.

Let $u=R(z) f$ be as in Theorem 7.4. Then it follows from (7.23) and the equation $-\Delta u-\mu(x) z u=\mu(x) f$ that

$$
\|u\|_{2,-\delta} \leq C\|f\|_{\delta}
$$

with $C=C(c, d, \mu, \delta)$. As in $\S 5$, the operator norm in $\mathbf{B}\left(H_{s}^{j}\left(\mathbf{R}^{2}\right), H_{t}^{\ell}\left(\mathbf{R}^{2}\right)\right)$ will be denoted by \|\|$_{(j, s)}^{(\ell, t)}$, where $j, \ell=0,1,2, s, t \in \mathbf{R}$. Thus we have

Theorem 7.6. Suppose that Assumption 2.1 holds with $N=2$. Let $1 / 2<\delta \leq 1$. Let $R(z)$ be the resolvent of $H$. Let $0<c<d<\infty$ and let $J_{ \pm}(c, d)$ be as above. Then there is a positive constant $C=C(c, d, \mu, \delta)$ such that

$$
\|R(z)\|_{(0, \delta)}^{(2,-\delta)} \leq C
$$

for $z \in J_{+}(c, d) \cup J_{-}(c, d)$.

Now we can proceed as in $\S 6$ to obtain the limiting absorption principle for $H$ with $N=2$.

Theorem 7.7. Suppose that Assumption 2.1 holds with $N=2$. Let $1 / 2<\delta \leq 1$. Let $R(z)$ be the resolvent of $H$.

(i) Let $\lambda>0$. Then the extended resolvent $R_{ \pm}(\lambda)$ is well-defined by

$$
R_{ \pm}(\lambda)=\lim _{\eta \downarrow 0} R(\lambda \pm i \eta)
$$

in $\mathbf{B}\left(L_{2, \delta}\left(\mathbf{R}^{2}\right), H_{-\delta}^{2}\left(\mathbf{R}^{2}\right)\right)$. 
(ii) Let $D_{ \pm}$be given by (6.6) and extend $R(z)$ on $D_{+}$as in (6.7), i.e.,

$$
R(\lambda+i \eta)= \begin{cases}R(\lambda+i \eta) & (\lambda>0, \eta>0), \\ R_{+}(\lambda) & (\lambda>0, \eta=0) .\end{cases}
$$

Extend $R(z)$ on $D_{-}$as in (6.8). Then $R(z)$ is a $\mathbf{B}\left(L_{2, \delta}\left(\mathbf{R}^{2}\right), H_{-\delta}^{2}\left(\mathbf{R}^{2}\right)\right)$ valued continuoius function on each of $D_{+}$and $D_{-}$.

(iii) For any $z \in D_{+}\left[\right.$or $\left.D_{-}\right], R(z)$ is a compact operator from $L_{2, \delta}\left(\mathbf{R}^{2}\right)$ into $H_{-\delta}^{1}\left(\mathbf{R}^{2}\right)$.

(iv) For $0<c<d<\infty$ there exists a constant $C=C\left(c, d, \delta, m_{0}, M_{0}\right)>$ 0 such that, for $z \in \bar{J}_{+}(c, d) \cup \bar{J}_{-}(c, d)$,

$$
\left\{\begin{array}{c}
\int_{E_{s}}(1+r)^{-2 \delta}\left(|\nabla R(z) f|^{2}+|k|^{2}|R(z) f|^{2}\right) d x \\
\leq C^{2}(1+s)^{-(2 \delta-1)}\|f\|_{\delta}^{2} \\
\left(s \geq 1, f \in L_{2, \delta}\left(\mathbf{R}^{N}\right)\right), \\
\|\mathcal{D} R(z) f\|_{\delta-1} \leq C\|f\|_{\delta} \quad\left(f \in L_{2, \delta}\left(\mathbf{R}^{N}\right)\right) .
\end{array}\right.
$$

Remark 7.8. In the case that $N \geq 3$, all the constants $C$ which appear in the evaluation of $R(z)$ are constructive, i.e., these constants $C=$ $C(c, d, \mu, \delta, \cdots)$ can be computed explicitly when the values of $c, d, \mu, \delta$ $\cdots$ are given. On the other hand, the constant $C$ in Proposition 7.5 is not constructive in our method, and hence the constant $C$ in Theorem 7.6 is not constructive, too.

\section{References}

[1] S. Agmon, Spectral properties of Schrödinger operators and scattering theory, Ann. Scuola Sup. Pisa 2 (1975), 151-218.

[2] M. Ben-Artzi, Y. Dermanjian and J.-C. Guillot, Acoustic waves in perturbed stratified fluids: a spectral theory, Commun. Partial Differential Equations 14 (1989), 479-517.

[3] A. Boutet de Monvel-Berthier and D, Manda, Spectral and scattering theory for wave propagation in perturbed stratified media, Universität Bielefeld, BiBos, preprint, Nr. 606/11/93. 
[4] S. DeBiévre and D. W. Pravica, Spectral analysis for optical fibres and stratified fluids I: The liming absorption principle, J. Functional Analysis $\mathbf{9 8}$ (1991) 406-436.

[5] S. DeBiévre and D. W. Pravica, Spectral analysis for optical fibres and stratified fluids II: Absence of eigenvalues, Commun. Partial Differential Equations 17, (1992), 69-97.

[6] D. Eidus, The limiting absorption and amplitude problems for the diffraction problem with two unbounded media, Comm. Math. Phys. 107 (1986), 29-38.

[7] T. Ikebe and Y. Saitō, Limiting absorption method and absolute continuity for the Schrödinger operator, J. Math. Kyoto Univ. 12 (1972), 513-542.

[8] W. Jäger and Y. Saitō, The limiting absorption principle for the reduced wave operator with cylindrical discontinuity, Preprint. IWR (SFB359) 94-74, University of Heidelberg. 1994.

[9] W. Jäger and Y. Saitō, The limiting absorption principle for the reduced wave operator with multimedia. Preprint.1995.

[10] G. Roach and B. Zhang, On Sommerfeld radiation conditions for the diffraction problem with two unbounded media, Proc. Royal Soc. Edinburgh 121A (1992), 149-161.

[11] Y. Saitō, The principle of limiting absorption for second-order differential equations with operator-valued coefficients, Publ. RIMS, Kyoto Univ. 7 (1972), 518-619.

[12] Y. Saitō, The principle of limiting absorption for the non-selfadjoint Schrödinger operators in $\mathbf{R}^{N}$ ( $N \neq 2$ ), Publ. RIMS, Kyoto Univ. 9 (1974), 397-428.

[13] Y. Saitō, A remark on the limiting absorption principle for the reduced wave equation with two unbounded media Pacific J. Math. 136 (1989), 183208.

[14] R. Weder, Absence of eigenvalues of the acoustic propagators in deformed waveguides, Rocky Mountain J. Math. 18 (1988), 495-503.

[15] R. Weder, Spectral and Scattering Theory for Wave Propagation in Perturbed Stratified Media, Springer-Verlaga, Berlin, 1991.

[16] C. Wilcox, Sound Propagation in Stratified Fluids, Springer-Verlag, New York, 1984.

[17] B. Zhang, On radiation conditions for acoustic propagators in perturbed 
stratified fluids. Preprint. 1994. . 